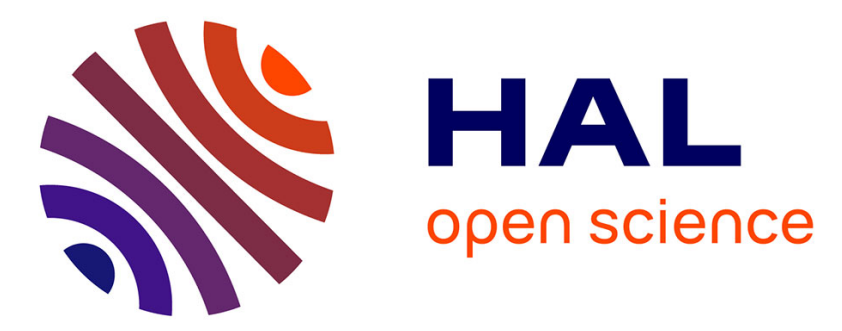

\title{
Breakdown and buildup mechanisms of cellulose nanocrystal suspensions under shear and upon relaxation probed by SAXS and SALS
}

Frédéric Pignon, Mathilde Challamel, Antoine de Geyer, Mohamad Elchamaa, Enrico Semeraro, Nicolas Hengl, Bruno Jean, Jean-Luc Putaux, Erwan Gicquel, Julien Bras, et al.

\section{To cite this version:}

Frédéric Pignon, Mathilde Challamel, Antoine de Geyer, Mohamad Elchamaa, Enrico Semeraro, et al.. Breakdown and buildup mechanisms of cellulose nanocrystal suspensions under shear and upon relaxation probed by SAXS and SALS. Carbohydrate Polymers, 2021, 260, pp.117751. 10.1016/j.carbpol.2021.117751 . hal-03148432

\section{HAL Id: hal-03148432 https://hal.science/hal-03148432}

Submitted on 30 Sep 2021

HAL is a multi-disciplinary open access archive for the deposit and dissemination of scientific research documents, whether they are published or not. The documents may come from teaching and research institutions in France or abroad, or from public or private research centers.
L'archive ouverte pluridisciplinaire HAL, est destinée au dépôt et à la diffusion de documents scientifiques de niveau recherche, publiés ou non, émanant des établissements d'enseignement et de recherche français ou étrangers, des laboratoires publics ou privés. 


\title{
Breakdown and buildup mechanisms of cellulose
}

\author{
nanocrystal suspensions under shear and upon \\ relaxation probed by SAXS and SALS
}

Frédéric Pignon $^{1 *}$, Mathilde Challamel ${ }^{1}$, Antoine De Geyer ${ }^{l}$, Mohamad Elchamaa $^{1}$, Enrico F.

Semeraro $^{1}$, Nicolas Hengl ${ }^{1}$, Bruno Jean ${ }^{2}$, Jean-Luc Putaux $^{2}$, Erwan Gicquel $^{3}$, Julien Bras $^{3}$, Sylvain Prevost ${ }^{4}$, Michael Sztucki ${ }^{4}$, Theyencheri Narayanan ${ }^{4}$, Henda Djeridi $^{5}$

* Corresponding author: frederic.pignon@univ-grenoble-alpes.fr

${ }^{1}$ Univ. Grenoble Alpes, CNRS, Grenoble INP (Institute of Engineering Univ. Grenoble Alpes), LRP, F38000 Grenoble, France

${ }^{2}$ Univ. Grenoble Alpes, CNRS, CERMAV, F-38000 Grenoble, France

${ }^{3}$ Univ. Grenoble Alpes, CNRS, Grenoble INP (Institute of Engineering Univ. Grenoble Alpes), LGP2, 38000 Grenoble, France

${ }^{4}$ ESRF, The European Synchrotron, CS 40220, F-38043 Grenoble Cedex 9, France

${ }^{5}$ Univ. Grenoble Alpes, CNRS, Grenoble INP (Institute of Engineering Univ. Grenoble Alpes), LEGI, 38000 Grenoble, France 


\title{
Highlights
}

- The structure of CNC suspensions under shear was studied by rheo-SAXS and SALS.

- A broad range of lengthscales from nanometer to micrometer has been explored.

- Changes in cholesteric organization and disappearance of pitch under shear have been evidenced.

- The CNC organization over the three-regime rheological behavior has been clarified.

- Buildup mechanisms, from nematic to cholesteric were highlighted on large scales.

\section{Graphical Abstract}

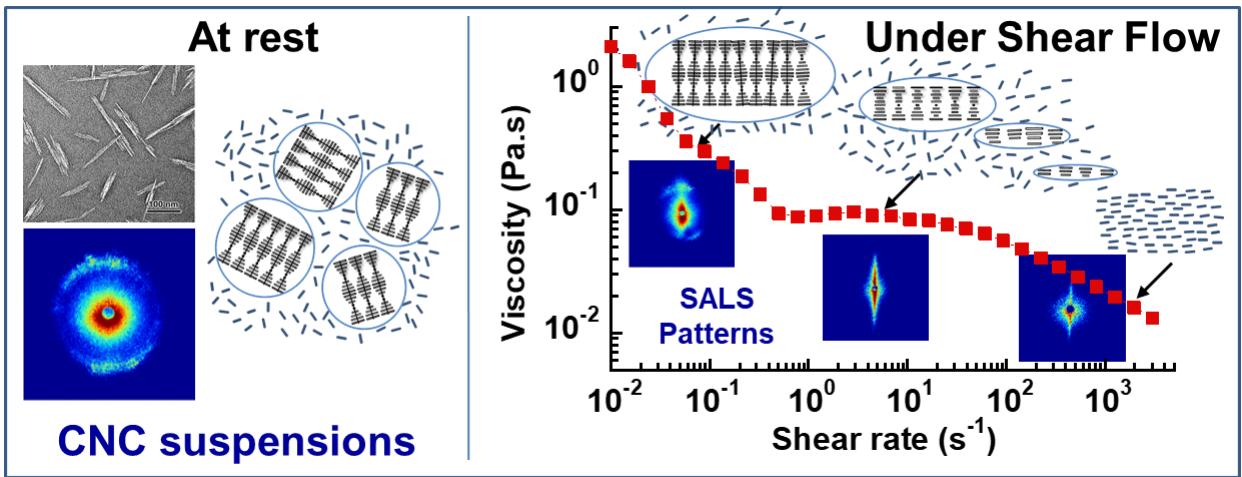

\begin{abstract}
The breakdown and buildup mechanisms in concentrated cellulose nanocrystal (CNC) suspensions under shear and during relaxation upon cessation of shear were accessed by smallangle X-ray or light scattering combined with rheometry. The dynamic structural changes over nanometer to micrometer lengthscales were related to the well-known three-regime rheological behavior. In the shear-thinning regime I, the large liquid crystalline domains were progressively fragmented into micrometer-sized tactoids, with their cholesteric axis aligned perpendicular to the flow direction. The viscosity plateau of regime II was associated to a further disruption into submicrometer-sized elongated tactoids oriented along the velocity direction. At high shear rate,
\end{abstract}


regime III corresponded to the parallel flow of individual CNCs along the velocity direction. Upon cessation of flow, the relaxation process occurred through a three-step buildup mechanisms: i) a fast reassembling of the individual $\mathrm{CNCs}$ into a nematic-like organization established up to micrometer lengthscales, ii) a slower formation of oriented large cholesteric domains, and iii) their isotropic redistribution.

Keywords: Cellulose nanocrystals, rheo-SAXS, SALS, shear-flow, cholesteric phase, structural organization.

\section{1. Introduction}

2 Cellulose nanocrystals $(\mathrm{CNCs})$ are particularly attractive crystalline nanoparticles for the design

3 of new bio-based materials with enhanced mechanical, optical or barrier properties. These

4 nanocelluloses display outstanding mechanical properties, such as shear modulus as high as 120 -

$5150 \mathrm{GPa}$ and strength up to $6 \mathrm{GPa}$ for CNCs (Eichhorn et al., 2005) together with high aspect ratio

6 and low density, in addition to their biodegradability, non-toxicity and biocompatibility (Siquieira

7 et al., 2010; Dufresne et al., 2017). The knowledge of the flow-induced structures in aqueous CNC

8 suspensions is crucial in their processing to bio-based materials with better performance and

9 controlled structural organization (Thomas et al., 2018). Moreover, understanding the complex

10 rheological behavior observed in these suspensions is of primary interest in the field of biorefinery.

11 More specifically, controlling the dynamic orientation of CNCs under flow has a direct

12 consequence on the macroscopic optical and mechanical properties of the material (Siquieira et al.,

13 2010; Dufresne et al., 2017; Kargarzadeh et al., 2018) and potential industrial applications

14 (Domingues et al., 2014; Tang et al., 2017). In order to improve the development of nanocellulose-

15 based materials, several processes involving shear flow or pressure forces have been subject of in 
16 situ structural investigations. For example, time-resolved in situ small-angle X-ray scattering 17 (SAXS) was successfully employed to access the structural organization and growth of 18 accumulated particles near the membrane surface during cross-flow ultrafiltration (Jin et al., 2015;

19 Rey et al., 2019; Semeraro et al 2020). In situ and real time grazing-incidence wide-angle X-ray scattering (GIWAXS) was used to investigate the influence of suspension concentration, shear-cast

21 velocity, and drying temperature on the ordering of the CNCs during the casting process (Sanchez-

22 Botero et al., 2018). To develop these processing methodologies and understand the effect of shear

23 flow on the structural arrangement in CNC suspensions, more fundamental approaches are 24 required.

25 In the literature, the structural organization in CNC suspensions at rest and their phase diagram 26 have been well characterized. CNC suspensions are known to exhibit a phase separation behavior 27 with coexisting isotropic and liquid-crystalline phases (Revol et al., 1992; Dong et al., 1996; Orts 28 et al., 1998; Ebeling et al., 1999; Schütz et al., 2015; Urena-Benavides et al., 2011; Lagerwall et 29 al., 2014; Lenfant et al., 2017; Gicquel et al., 2019; Xu et al., 2020). At rest, these two phases 30 macroscopically separate over time since the liquid-crystalline phase has a higher density. This 31 lower phase, referred to as the chiral nematic or cholesteric phase, is characterized by a long-range 32 orientational order of the $\mathrm{CNCs}$ combined with a helical modulation of their director (local 33 alignment) (Lagerwall et al., 2014; Mitov, 2012). Before complete phase separation, the cholesteric 34 phase exists as microdomains, or tactoids, in the isotropic phase (Wang et al., 2016; Wang and 35 MacLachlan 2018; Tran et al., 2018; Liu et al., 2019). The phase diagram is characterized by two 36 critical concentrations, $C_{i}$, and $C_{a}$. Below $C_{i}$, the suspension is fully isotropic and above $C_{a}$ it is 37 fully anisotropic (cholesteric). The coexistence region lies between these two values. The structural 38 features of this phase separation have been investigated by different techniques. Firstly, at 
39 nanometric lengthscales, SAXS and small-angle neutron scattering (SANS) measurements have

40 allowed the determination of the evolution of the interparticle distance, $d$, between CNCs as a

41 function of concentration. Secondly, at micrometric scale, polarized light optical microscopy was

42 used to determine the phase diagram and measure the helical pitch, $P$, as a function of volume

43 fraction (Revol et al., 1992; Bercea and Navard 2000; Dong et al., 1996, Urena-Benavides et al.,

44 2011). $P$ corresponds to the distance needed for a $360^{\circ}$ rotation of the director, which can also be

45 obtained from laser diffraction experiments (Schütz et al., 2015). These results have shown that

46 both $d$ and $P$ decrease with increasing concentration.

47 The rheological behavior of CNC suspensions under shear and at intermediate concentrations, 48 corresponding to the co-existence of isotropic and cholesteric phases, displays the well-known

49 three-region behavior typical of liquid crystalline systems, characterized by a shear-thinning region

50 followed by plateau region and then a second shear-thinning region (Onogi and Asada, 1980).

51 Aiming at understanding this complex rheological behavior, some previous studies focused on

52 characterizing the organization of CNCs under shear. First, at nanometer lengthscales, for example

53 by rheo-SANS (Orts et al., 1998; Haywood et al., 2017) or rheo-SAXS (Ebeling et al., 1999), and

54 second at micrometric lengthscales by polarized light optical microscopy (Bercea and Navard

55 2000; Shafiei-Sabet et al., 2012; 2014; Derakhshandeh et al., 2013), as well as rheo-SALS (small-

56 angle light scattering) (Xu et al., 2017; Qi et al., 2019) and single droplet oscillatory platform with

57 fluorescence and polarized light optical microscopy (Alizadehgiashi et al., 2018). These results

58 enabled structural interpretation of the three-domain regime. The first region of shear thinning has

59 been attributed to a progressive disruption of large chiral nematic domains into smaller ones. The

60 plateau region corresponds to the increasing alignment of these small chiral domains along the flow

61 direction with their helical axis orienting perpendicular to the velocity direction. Finally, the second 
62 region of shear thinning at the highest shear rates corresponds to the disruption of all these

63 cholesteric domains, releasing all CNCs that, in turn, could be individually oriented along the flow

64 direction. Nevertheless, until now, only few results have been obtained at the micrometer scale,

65 which would be the relevant lengthscale to follow the evolution of the cholesteric organization

66 under shear. Polarized light optical observations under shear (Shafiei-Sabet al., 2012;

67 Derakhshandeh et al., 2013; Shafiei-Sabet et al., 2014) have shown the breakdown and alignment

68 of chiral nematic domains pertaining to the three-region behavior, and rheo-SALS data have

69 revealed the shear-induced breakup of the CNCs above a critical shear rate (Xu et al., 2017) or

70 under different $\mathrm{pH}$ conditions (Qi et al., 2019). Studying film formation by polarized light

71 microscopy upon drying of CNC suspensions, Gray and Mu (Gray and Mu, 2019) attempted to

72 decouple changes in texture due to evaporation from those due to shear relaxation for a sample in

73 which gelation occurred before it reached the iridescence pitch values. These authors observed a

74 transitory nematic-like texture that was induced by shear during the preparation of the sample for

75 polarized light microscopy. They proposed that the transition between chiral nematic and nematic

76 structures involved a twist-bend-like intermediate and not an untwisting of the chiral nematic phase

77 (Gray and Mu, 2016). From polarized light optical and scanning electron microscopy images, Park

78 et al. (2014) have shown that a better control of the helix orientation was achieved in films of dried

79 CNCs by subjecting the suspension to a circular shear flow during drying. This induced the

80 deformation of tactoids into ellipsoids, thereby breaking the symmetry in such a way that a vertical

81 helix orientation became favored.

82

83 Despite all these recent advances on the monitoring of the organization of CNC suspensions,

84 until now, no in situ characterization of the pitch evolution at the micrometric lengthscale as a

85 function of the shear flow has been carried out. In this work, in order to obtain a complete structural 
86 interpretation of this complex three-regime flow curve, the mechanical response under flow

87 (viscosity as a function of shear rate) has been correlated to structural observations at different

88 lengthscales: from the nanometer scale of the CNCs to the micrometer scale corresponding to their

89 organization into a chiral nematic liquid-crystalline phase.

90 New features of the organization of CNCs at micrometer lengthscales were evidenced thanks to

91 dedicated channel-type quartz flow cells combined with a 2D-SALS set-up (Piau et al., 1999).

92 Specifically and unambiguously, this allowed characterizing the evolution of the pitch distance and

93 its appearance or disappearance as a function of time and applied shear rate both under flow and

94 upon its cessation. Furthermore, a combined rheology and small-angle X-ray scattering (rheo-

95 SAXS) setup available at the European Synchrotron Radiation Facility (ID02 TRUSAXS

96 beamline) allowed an in situ and time-resolved study of the CNC orientations and structural

97 properties at nanometer scales in the dispersions under flow. More specifically, new aspects have

98 been evidenced thanks to measurements performed in radial and tangential modes of observation.

99 From in situ SALS and SAXS measurements, a straightforward analysis of the 2D-patterns with

100 azimuthal, sector or annular average of the scattering intensities over a broad $q$-range allowed to

101 deduce the time dependent evolutions of three important parameters: the anisotropy parameter, the 102 mean orientation direction of flowing objects, and the pitch value, $P$ as well as its presence or

103 disappearance. Correlated with the changes in viscosity measurements, the evolution of these three

104 parameters as a function of shear rate and time enabled a consistent description of the breakdown

105 and buildup mechanisms of the structural organization of CNCs from nanometer to micrometer

106 lengthscales. The dynamic changes of liquid crystalline microdomains, or tactoids, and their chiral

107 nematic organization under shear flow were then related to the well-known three-regime behavior,

108 as well as during the relaxation after cessation of shear. 


\section{2. Materials and Methods}

$111 \quad$ 2.1. Cellulose Nanocrystals

112 Cellulose nanocrystals (CNCs) were purchased from UMaine Development Center (University 113 of Maine, USA) as an aqueous suspension with a stock concentration of $C=12.2 \mathrm{wt} \%$. A series of

114 samples at different concentrations were prepared by dilution in deionized water followed by $2 \mathrm{~h}$

115 of vigorous stirring of the suspensions. $\mathrm{NaCl}$ was added to reach a fixed ionic strength of $10^{-2}$ $116 \mathrm{~mol} \mathrm{~L}{ }^{-1}$. These suspensions were then sonicated using a sonication probe (Branson Digital sonifier) 117 and applying at least $2 \mathrm{~kJ}$ per gram of CNCs.

119 2.2. Rheo-SAXS

120 All X-ray scattering measurements were performed at the ID02 beamline at the European 121 Synchrotron Radiation Facility (ESRF), Grenoble, France (Narayanan et al., 2018). The 122 wavelength of incident X-rays was $\lambda=0.995 \AA$ and two sample-to-detector distances $D(2$ and 10 $123 \mathrm{~m})$ were used. SAXS measurements covered the range of scattering vector magnitude: $8 \times 10^{-3} \mathrm{~nm}^{-}$

$124{ }^{1} \leq q \leq 5 \mathrm{~nm}^{-1}$, where $q=(4 \pi / \lambda) \sin (\theta / 2)$ with $\theta$ the scattering angle. The corresponding nominal 125 lengthscale $(\sim 2 \pi / q)$ range is $1.25 \mathrm{~nm} \leq 1 \leq 785 \mathrm{~nm}$. Combined rheometric and SAXS measurements 126 were carried out using the Rheo-SAXS set-up available at the ESRF (Panine et al., 2003). The 127 setup consisted of a Haake Rheo-Stress RS6000 stress-controlled rheometer, and homemade 128 polycarbonate Couette-type shear cells with internal diameter ID $=20 \mathrm{~mm}$, outer diameter OD = $12921.5 \mathrm{~mm}$, and height $=50 \mathrm{~mm}$. The bottom gap was $0.6 \mathrm{~mm}$ in order to avoid any artefact from 130 boundary conditions. The temperature was set to $25^{\circ} \mathrm{C}$ with a stability of $0.1{ }^{\circ} \mathrm{C}$. Both radial and 131 tangential positions were used to access different observation planes and allowed discriminating a 
132 uniaxial or probable biaxial CNC orientation. The incident X-ray beam crossed through either the

133 center of the cell along the radial direction $(\mathrm{Ra})$ (perpendicular to the velocity/vorticity plane) or

134 the center of the gap along the tangential direction (Ta) (perpendicular to vorticity/shear gradient

135 plane) where $v$ is the velocity and grad $v$ is the velocity gradient (Panine et al., 2003). In the radial

136 configuration $(\mathrm{Ra})$, the beam was parallel to the velocity gradient $(\operatorname{grad} v)$, and the $(z, v)$ plane was

137 probed. In the tangential position (Ta), the beam was parallel to the velocity direction $(v)$, and the

$138(z, \operatorname{grad} v)$ plane was probed as shown in Fig. 1. For this reason, the beam size was collimated to

$1390.3 \mathrm{~mm} \times 0.15 \mathrm{~mm}$ in the vertical and horizontal directions, respectively. Measured scattering 140 patterns were normalized to an absolute intensity scale after applying standard detector corrections

141 and subsequently azimuthally averaged to yield $\mathrm{I}(q)$, or sector averaged in vertical $z$ direction, to

142 yield $\mathrm{I}_{\mathrm{v}}(q)$. Before the shear flow experiments with CNC suspensions, the Couette-type shear-cell

143 was filled with demineralized water (Milli-Q) and the normalized background scattering of the cell

144 was registered. This background scattering of the cell filled with water was subtracted from the

145 normalized scattering patterns of the CNC suspensions in all shear flow experiments. For static 146 sample characterization, a temperature-controlled flow-through capillary cell (inner diameter = $1471.95 \mathrm{~mm}$ ) was used to measure the absolute scattered intensity of dispersions at rest at $\mathrm{T}=22{ }^{\circ} \mathrm{C}$.

148 It allowed measuring sample and background scattering at the same position of the capillary, 149 thereby allowing a very reliable subtraction.

\subsection{SALS under Shear Flow}

152 The light scattering set-up used for the experiment was developed and built as described 153 elsewhere and schematically shown in Fig. 2 (Piau et al., 1999). It consists of a $2 \mathrm{~mW}$ laser beam 154 (He-Ne) with a wavelength of $632.8 \mathrm{~nm}$ and a Fresnel lens for collection of the forward scattered 
155 light. The detector was an Allied Vision (AV MAKO G-419B POE) monochrome digital camera:

156 CMOS sensor $\left(2048 \times 2048\right.$ pixels, $\left.11.3 \times 11.3 \mathrm{~mm}^{2}\right)$. Image processing was performed with a

157 video system and specifically developed software (Vimba Matlab) that allowed regrouping and 158 averaging of scattering intensity with SAXS Utilities software (Sztucki and Narayanan 2007). The

159 scattering patterns were recorded by the camera and video system throughout the experiment. The

160 modulus of the scattering vector is given by $q=[(4 \pi / \lambda) \sin (\theta / 2)]$, where $\lambda$ is the wavelength of the

161 light in the sample and $\theta$ the scattering angle. The light scattering measurements covered a $q$-range

162 from $2 \times 10^{-4}$ to $4 \times 10^{-3} \mathrm{~nm}^{-1}$. The shear flow cell consisted of a rectangular quartz glass cell

163 (height $\mathrm{H}=1 \mathrm{~mm}$, width $\mathrm{W}=7.4 \mathrm{~mm}$, and length $\mathrm{L}=10 \mathrm{~mm}$ ) as depicted in Fig. 2 (these two

164 dimensions have been chosen to fit with usual convention, although the channel in this case is

165 positioned vertically (with the longer dimension in vertical direction) contrary to the usual usage

166 for example in microscopy observations where the channel is positioned horizontally.

167 The shear flow cell was coupled with a syringe pump to vary the flow rate $Q$ and thus the shear 168 rate inside the channel. For high aspect ratio $\mathrm{H} / \mathrm{W} \ll 1$ which is the case here $\mathrm{H} / \mathrm{W}=0.135$, the 169 mean shear rate $\dot{\gamma_{y}}$ in the rectangular channel was calculated using the following equation (Son, 170 2007).

$171 \quad \dot{\gamma}_{y}=\frac{6 Q}{W H^{2}} \quad$ (Eq. 1)

172 To evaluate the variation of shear rate inside the channel in the $x$ direction along the laser beam 173 propagation, in the thickness $\mathrm{H}$ of the sample, calculations were performed (Fig. S1) from (Son, 174 2007) of the apparent shear rate $\dot{\gamma_{a}}$ of a rectangular die and the wall shear rate $\dot{\gamma_{w}}$ that took into 175 account the shear thinning behavior (and corresponding shear thinning index $n$ for each regimes of 176 flow). This values of shear thinning index $n$ were deduced from our previous results (Gicquel et 177 al., 2019). One can see that for regimes II and III, the differences are negligible for the three 
178 calculated shear rates $\left(\dot{\gamma_{y}}, \dot{\gamma_{a}}, \dot{\gamma_{w}}\right)$ and that for regime I the wall shear rate $\dot{\gamma_{w}}$ which is higher for 179 each flow rate applied in regime I, stay in the limits of the regime I for the exploited results in this 180 work.

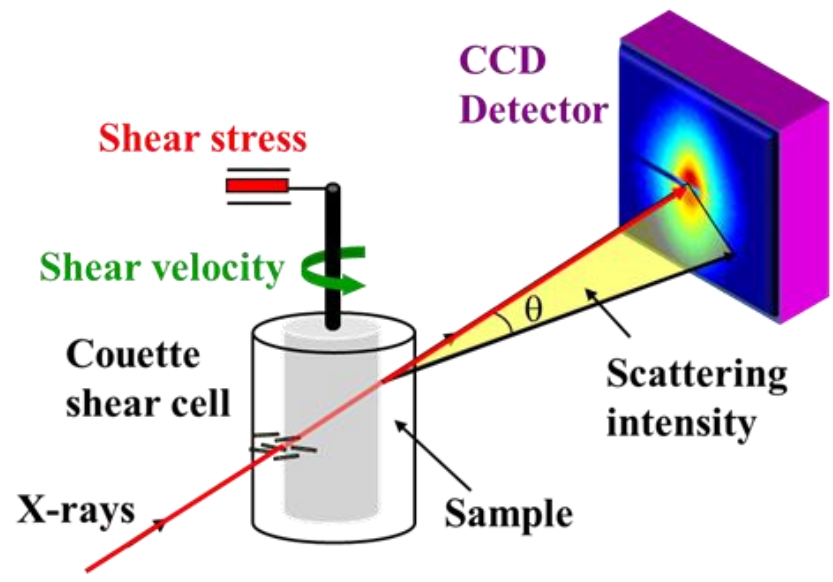

(Ra)

(Ta)

Radial Tangential

Position Position
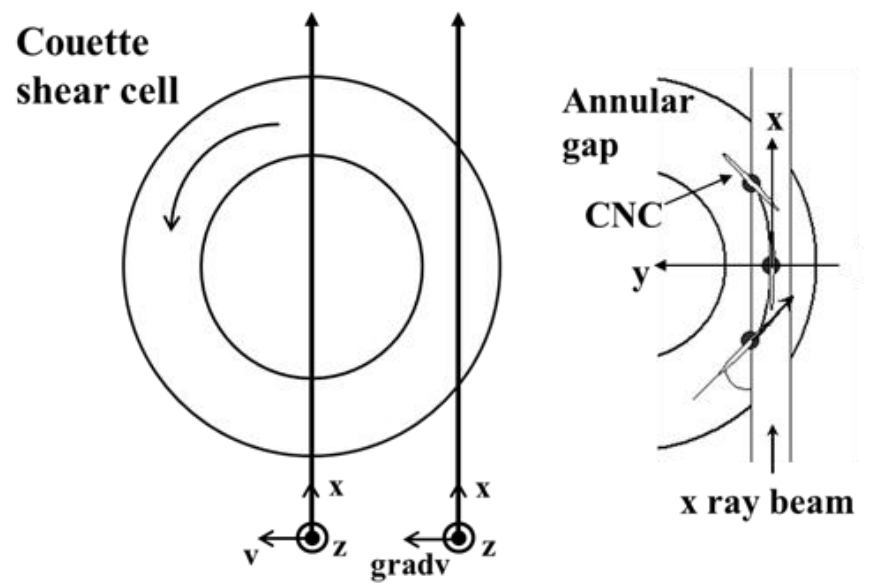

182

183 Figure 1. Rheo-SAXS setup and observation planes related to radial or tangential beam position.

The flow rate $Q$ values ranged from $1 \mu \mathrm{L} \mathrm{min}^{-1}$ up to $\cong 150000 \mu \mathrm{L} \mathrm{min} \operatorname{m}^{-1}\left(0.01 \mathrm{~s}^{-1}\right.$ to $\left.2027 \mathrm{~s}^{-1}\right)$.

186 During the experiments, the laser beam was directed perpendicular to the walls of the flow cell,

187 along the $x$-direction and is positioned in middle of the channel $(z=3.5 \mathrm{~mm})$, through the sample 
188 that scattered on the Fresnel lens. The scattering intensity collected by the Fresnel lens was then

189 focused towards the camera and recorded. The azimuthal averages of the scattering intensities were

190 calculated using the SAXS Utilities software. In order to establish the magnitude of anisotropy in

191 the vertical and horizontal directions, scattering along the vertical $\left(I_{z}\right)$ and horizontal $\left(I_{y}\right)$ axes were

192 extracted by integrating the scattering intensity over a sector of $20^{\circ}$ around the vertical $(z)$ and

193 horizontal ( $y$ ) axes, respectively. Two anisotropic degrees, $I_{v}$ and $I_{h}$, were defined as the mean value

194 of $I_{z}$ and $I_{y}$ in the $q$-range $(1.344-1.656) \times 10^{-3} \mathrm{~nm}^{-1}$. From these integrations the mean anisotropy

195 parameter was defined (Pignon et al., 2009):

196 Anisotropy SALS $=$ abs $\left(\left(I_{v}-I_{h}\right) /\left(I_{v}+I_{h}\right)\right) \quad$ (Eq. 2).

197 Annular averages were also calculated over $q$ range from $\left(2.10^{-3}\right.$ to $\left.4.10^{-3}\right) \mathrm{nm}^{-1}$, to define the

198 scattering intensity as a function of the $\psi$ azimuthal scattering angle in the plane of the 2D SALS

199 patterns, in order to quantify the degree of orientation as well as the angular variation of this degree

200 of orientation. It allowed following the maximum intensity of the $q_{\mathrm{P}}$ representative of the direction

201 of the helical axis of the cholesteric organization, or the orientation of the oval shaped SALS

202 patterns, related to the orientation of the nematic like tactoids, as well as the relaxation features.

203 Consequently, this set-up allowed accessing the orientation and structural changes in each CNC

204 suspension, as well as the pitch distance evolution, under flow or relaxation, as a function of time

205 and shear rate $\dot{\gamma_{y}}$. 


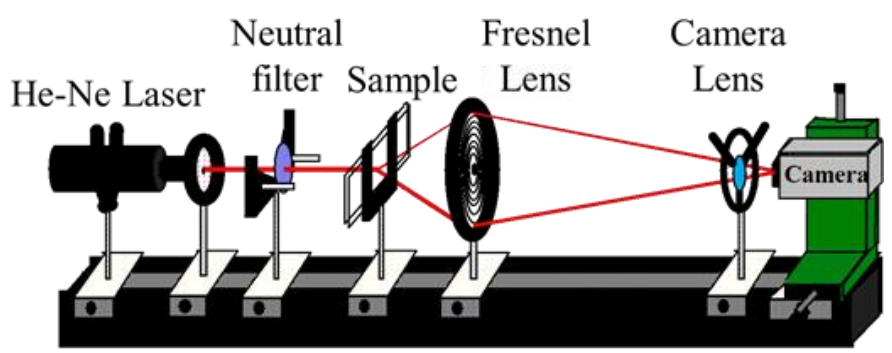

Shear Flow Cell

$\mathrm{H}=1 \mathrm{~mm} \mathrm{~W}=7.4 \mathrm{~mm} \mathrm{~L}=100 \mathrm{~mm}$
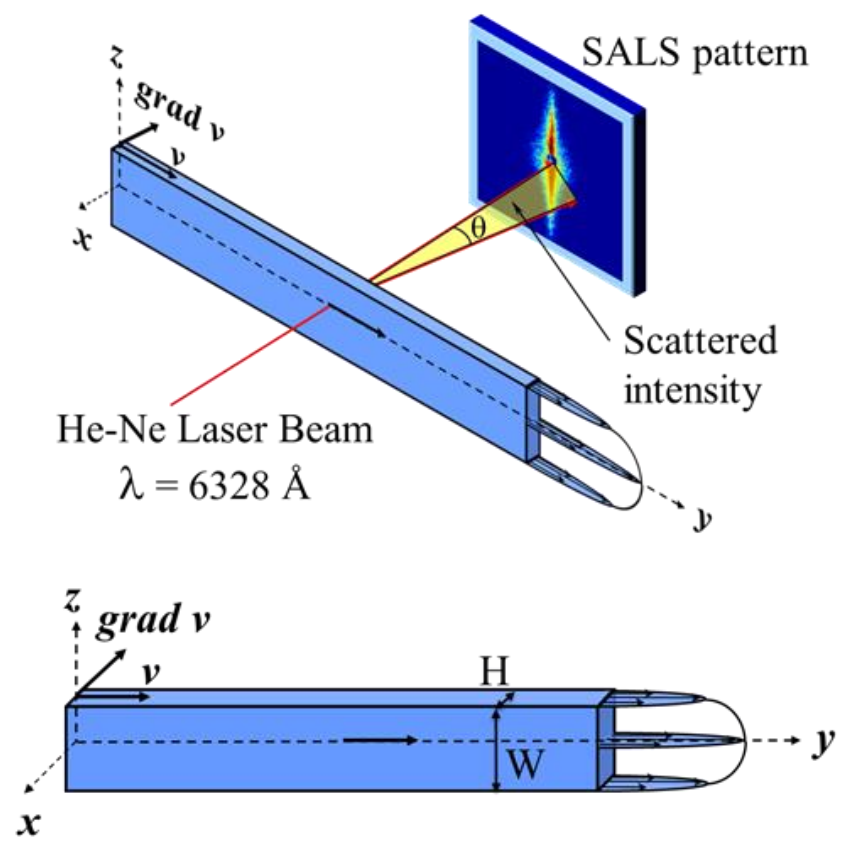

Figure 2. Schematic description of the SALS under shear flow setup.

\subsection{Rheometric Measurements}

210 Rheometric measurements were carried out using a shear rate controlled rheometer (DHR3, TA

211 Instrument) with a cone-plate geometry of $65 \mathrm{~mm}$ diameter and angle of $2^{\circ}$ in the same conditions

212 as in scattering experiments. These values of shear thinning index $n$ were deduced from our

213 previous results (Gicquel et al., 2019; Rey et al., 2019). Measurements were performed at a fixed

214 temperature of $25 \pm 1{ }^{\circ} \mathrm{C}$. An evaporation-proof system has been added on the top of the geometry

215 to ensure the saturation of the atmosphere of the sample. Direct observations of the shear profile at 
216 the outer edge of the rheometer tools, confirmed that slip did not occur and that the samples were

217 sheared uniformly without any localization phenomena.

\subsection{Transmission Electron Microscopy (TEM)}

220 Droplets of dilute CNC aqueous suspensions were deposited onto glow-discharged carbon221 coated copper grids and negatively stained with 2 wt $\%$ uranyl acetate. The specimens were 222 observed with a JEOL JEM-2100-Plus microscope operating at an accelerating voltage of $200 \mathrm{kV}$.

223 Images were recorded with a Gatan Rio 16 camera.

\section{Results and Discussions}

\subsection{Structure of CNC Suspensions at Rest and Phase Diagram}

The morphology of the CNCs considered in this work and their phase diagram in aqueous suspension have been studied in detail in previous publications (Gicquel et al., 2019; Semeraro et al., 2020). SAXS data from diluted suspensions at rest, assuming a parallelepipedal shape for the sonicated CNCs, gave $121 \times 20 \times 5 \mathrm{~nm}^{3}$ average dimensions. The TEM images showed individual acicular particles constituted of a few laterally-associated rodlike crystallites as presented in Fig. S2 (Gicquel et al., 2019). From previous polarized light optical observation of several suspensions at rest (Gicquel et al., 2019), three regions and two critical concentrations were described in the

234 phase diagram. The first critical concentration, $C_{i}$, defined the beginning of the biphasic regime 235 and had a value $C_{i} \sim 7.5 \mathrm{wt} \%\left(\phi_{i}=4.82 \mathrm{vol} \%\right)$ and the second critical concentration, $C_{a}$, 236 corresponded to the onset of the fully anisotropic phase with $C_{a} \sim 13.3 \mathrm{wt} \%$ ( $\phi_{a}=8.75 \mathrm{vol} \%$ ).

237 Below $C_{i}$, only the isotropic phase was present in the whole sample. The biphasic domain extended 238 from $C_{i}$ to $C_{a}$. In this intermediate domain, the volume fraction of the liquid-crystalline phase 
239 increased according to the regular increase of the chiral nematic domain. Above $C_{a}$, the anisotropic 240 phase occupies the whole volume of the sample.

241 Figure 3a shows the azimuthally-averaged scattering intensity, $I(q)$, of CNC suspensions plotted

242 over a broad $q$-range combining SAXS and SALS data of suspensions at rest. The mean 243 interparticle distance $d$ as well as the phase diagram of these sonicated CNC suspensions as a

244 function of CNC concentration have previously been studied (Gicquel et al., 2019; Semeraro et al.,

245 2020). The mean interparticle distance between the elementary CNCs has been deduced from the 246 scattering intensities of CNC suspensions probed by SAXS. I(q) vs $q$ plots of the SAXS data 247 revealed a maximum of intensity at a position $q_{\text {peak }}$ for each CNC concentration (Rey et al., 1999).

248 The mean interparticle distance $d$ was then calculated from $d=2 \pi / q_{\text {peak }}$ and plotted as a function of 249 concentration in Fig. 3b. As shown in the previous work (Gicquel et al., 2019) the power law fits 250 of the $d(C)$ evolution yielded a $d \propto C^{-1 / 2}$ scaling behavior, which is characteristic of long cylinders 251 or rod-like colloids in a nematic arrangement. Time resolved SAXS performed on a levitating CNC 252 drop have revealed structure evolutions of CNC dispersions during evaporation (Liu et al., 2019). 253 Scaling analysis of the concentration dependence of the separation distance suggested that 254 assembly of CNC can be divided into three regions as a function of the concentration with power 255 law exponents ranging from $-1 / 3$ to -1 with increasing concentration. Comparing the concentration 256 dependence of the interparticle distance measured in sealed capillaries, good agreement was found 257 for the scaling behavior, $d \propto C^{-1 / 2}$ for volume fractions between 2 and 6 vol $\%$ characteristic for 258 the biphasic state of CNC dispersions. 
a)

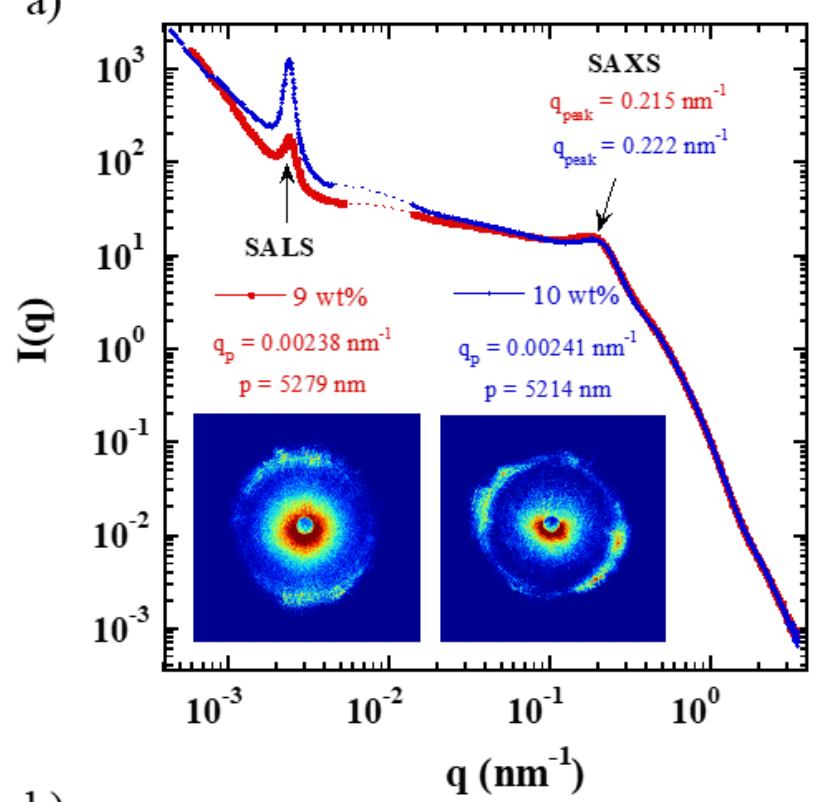

b)

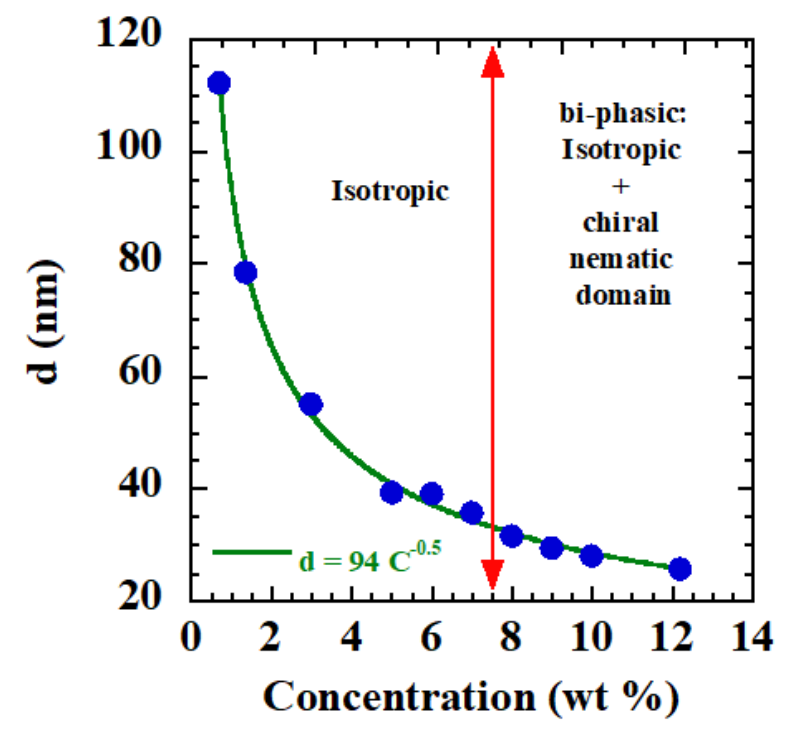

c)

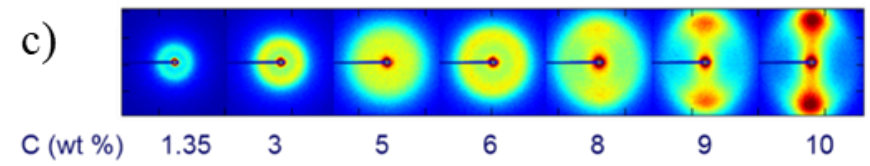

260 Figure 3. Scattered intensity versus $q$ plots from SAXS and SALS data of 9 and 10 wt\% CNC 261 suspensions at rest a) concentration dependence of the interparticle distance, $d$ of CNC suspensions 262 at rest. Pitch values, $P$, in (a) were deduced from the $q_{\text {P }}$ position in the SALS pattern and 263 interparticle distance, $d$ in (b) were deduced from the $q_{\text {peak }}$ position observed in the SAXS pattern 264 in (c). 
266 SALS measurements of suspensions at rest allowed characterizing the pitch of the cholesteric

267 phase for two CNC concentrations. The SALS patterns of suspensions at rest revealed a ring 268 corresponding to a particular distance in the sample as presented in Fig. S3. As described in several 269 previous analyses (Schütz et al., 2015; Frka-Petesic et al., 2017; Liu et al., 2019), this ring was 270 attributed to the half-pitch of the cholesteric phase. The pitch values $P$, were calculated from the $q_{\mathrm{P}}$ 271 position in the SALS patterns using $P=4 \pi / q_{\mathrm{P}}$, giving $P=5279$ and $5214 \mathrm{~nm}$ for CNC suspensions 272 at $9 \mathrm{wt} \%(\phi=5.82 \mathrm{vol} \%)$ and $10 \mathrm{wt} \%(\phi=6.49$ vol $\%)$ respectively.

\subsection{Procedures to Monitor Breakdown and Buildup Mechanisms}

275 Three procedures have been used to characterize the changes in structural organization under 276 shear or to follow relaxation processes. In the first procedure (P1), the CNC suspension was

277 injected in the canal at a large Q of $79 \mathrm{~L} \mathrm{~min}^{-1}$ corresponding to a shear rate of $1068 \mathrm{~s}^{-1}$. Then the 278 suspension was kept at rest for 2 to $3 \mathrm{~h}$ (rest step), until the cholesteric organization was recovered.

279 This was checked by monitoring the SALS patterns, which showed an isotropic diffuse scattering 280 (random orientation of the cholesteric phase in the canal) alongside a ring-like pattern related to 281 the presence of the pitch (Fig. S3). This rest step ensured that all the subsequent measurements 282 were starting from the same initial conditions, with no specific orientation and an anisotropic phase 283 volume fraction at its equilibrium value. Then, a specific constant shear rate (flow rate Q) was 284 applied and a series of SALS patterns were recorded to follow the transient state of the structural 285 organization as a function of time, up to reaching the corresponding steady state (stabilization of 286 SALS patterns). This P1 procedure was then performed at three shear rates corresponding to the 287 middle of regimes I, II and III. The second (P2) and third (P3) procedures were identical to P1 until 288 the rest step. Then, for P2, the shear rate was increased step by step, waiting for stabilization of the 
SALS pattern prior to applying the next shear rate value. Instead, in P3, a high shear rate was

290 applied until the stable state was reached, then the syringe pump was stopped and SALS patterns

291 were acquired to follow the relaxation of the structural organization as a function of time.

\subsection{Break-down Mechanisms of CNC Suspensions under Shear}

\subsubsection{Rheological Behavior in Steady-state}

295 First, rheometric measurements in cone-plate geometry were performed in order to assess the 296 typical three-regime behavior of these CNC suspensions. The steady-state shear viscosity as a

297 function of the shear rate applied has been obtained for different CNC concentrations within the 298 co-existence region of the phase diagram as presented in Fig. 4. The three-region behavior was

299 clearly observed. These measurements allowed to precisely define the critical shear rates separating 300 these three regimes for each CNC concentration (Orts et al., 1998; Shafiei-Sabet et al., 2012 - 2014 301 -2017; Derakhshandeh et al., 2013; Xu et al., 2016; Haywood et al., 2017; Lenfant et al., 2017;

302 Gicquel et al., 2019). As shown in Fig. 4, the extent of these three regimes was defined with 303 precision for the three highest concentrations from 8 to $10 \mathrm{wt} \%$ : the first shear-thinning regime (I)

304 at the lowest shear rates, the viscosity plateau regime (II), at the intermediate shear rates and the 305 second shear-thinning regime (III) at the highest shear rates. The shear rates applied in these 306 rheometric measurements have been reproduced in the canal type shear flow cell during in situ

307 SALS experiment in order to relate the viscosity features described in Fig. 4 to the structural 308 organization. Equation 1 was used to calculate the corresponding flow rate, $Q$, applied by the 309 syringe pump, in order to perform each shear flow experiment with the same shear rate $\dot{\gamma_{y}}$ as the 310 one used during conventional rheometric measurements. 

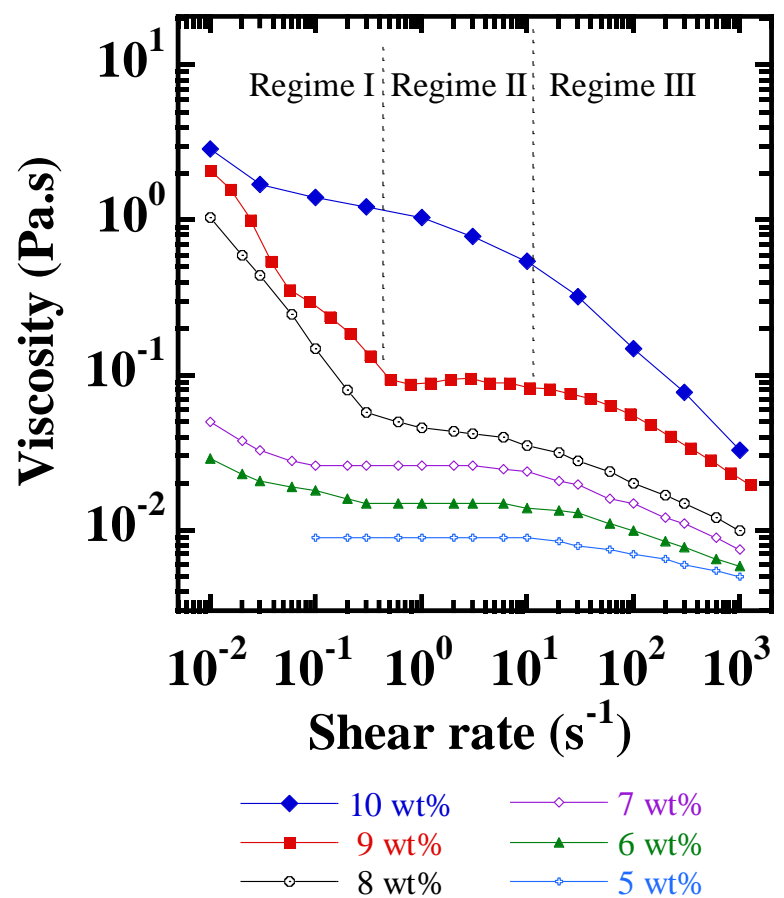

312 Figure 4. Steady-state flow curve (viscosity as a function of shear rate) of CNC suspensions at

313 different concentrations at $10^{-2} \mathrm{~mol} \mathrm{~L}^{-1} \mathrm{NaCl}$ and the evidence for the three-regime behavior.

314 Rheometric measurements were performed in cone-plate geometry at $T=24.5^{\circ} \mathrm{C}$.

316 3.3.2. Structural Organization at Micrometric Lengthscale: in situ SALS under Shear

317 Starting from a $9 \mathrm{wt} \%$ suspension after the rest step of P1, at $t=0 \mathrm{~s}$, SALS patterns consisted 318 of an isotropic ring-like pattern corresponding to a random orientation of the cholesteric

319 assemblies. For the three regimes, one shear rate pertaining to each regime was explored in details.

320 The corresponding SALS time evolution and their analysis by radial, vertical/horizontal sector 321 averaged or annular averaged integrations are presented in Fig. 5 a-c. 
a) Regime I: shear rate $=0.29 \mathrm{~s}^{-1}$
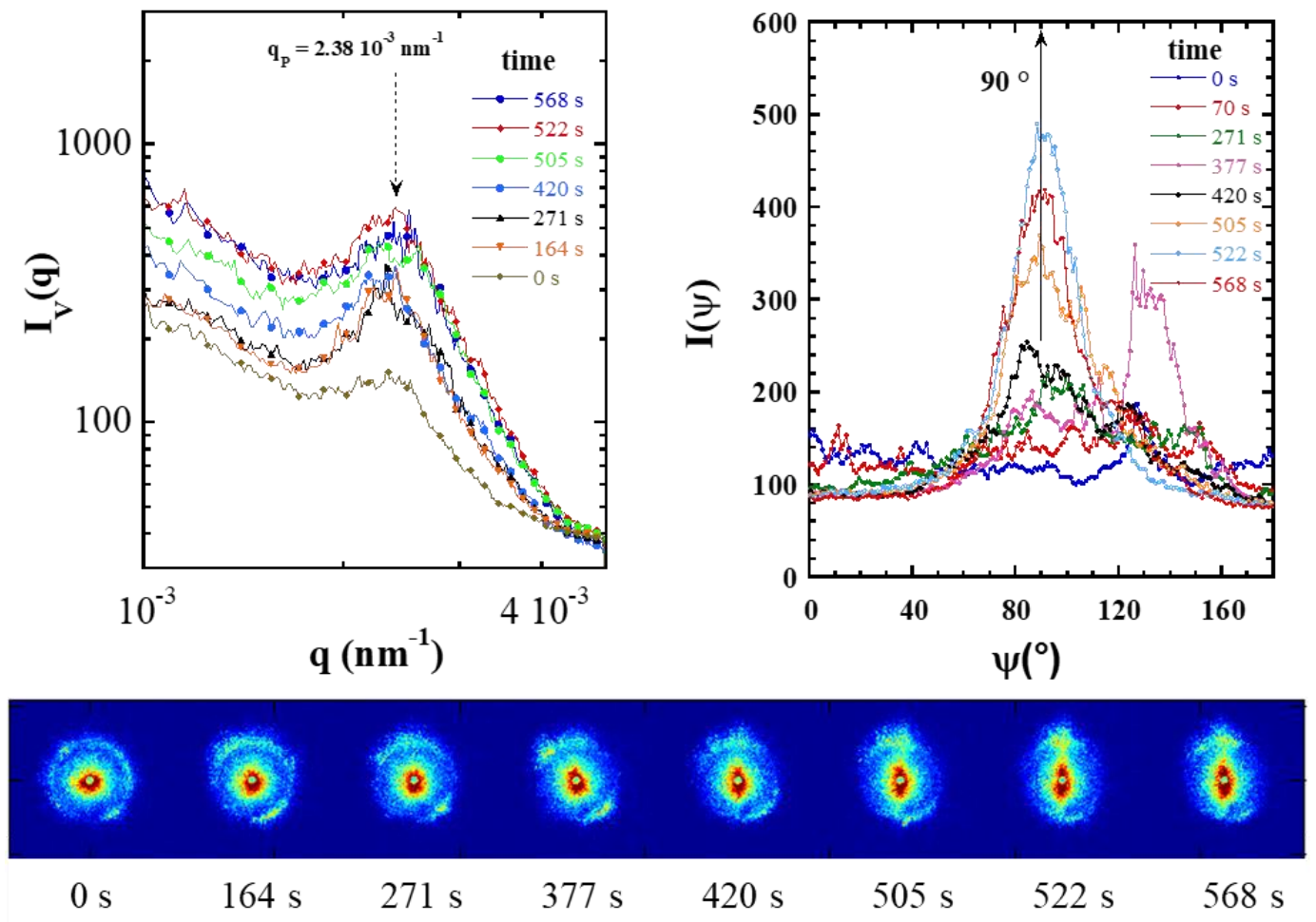

b) Regime II: shear rate $=13.3 \mathrm{~s}^{-1}$
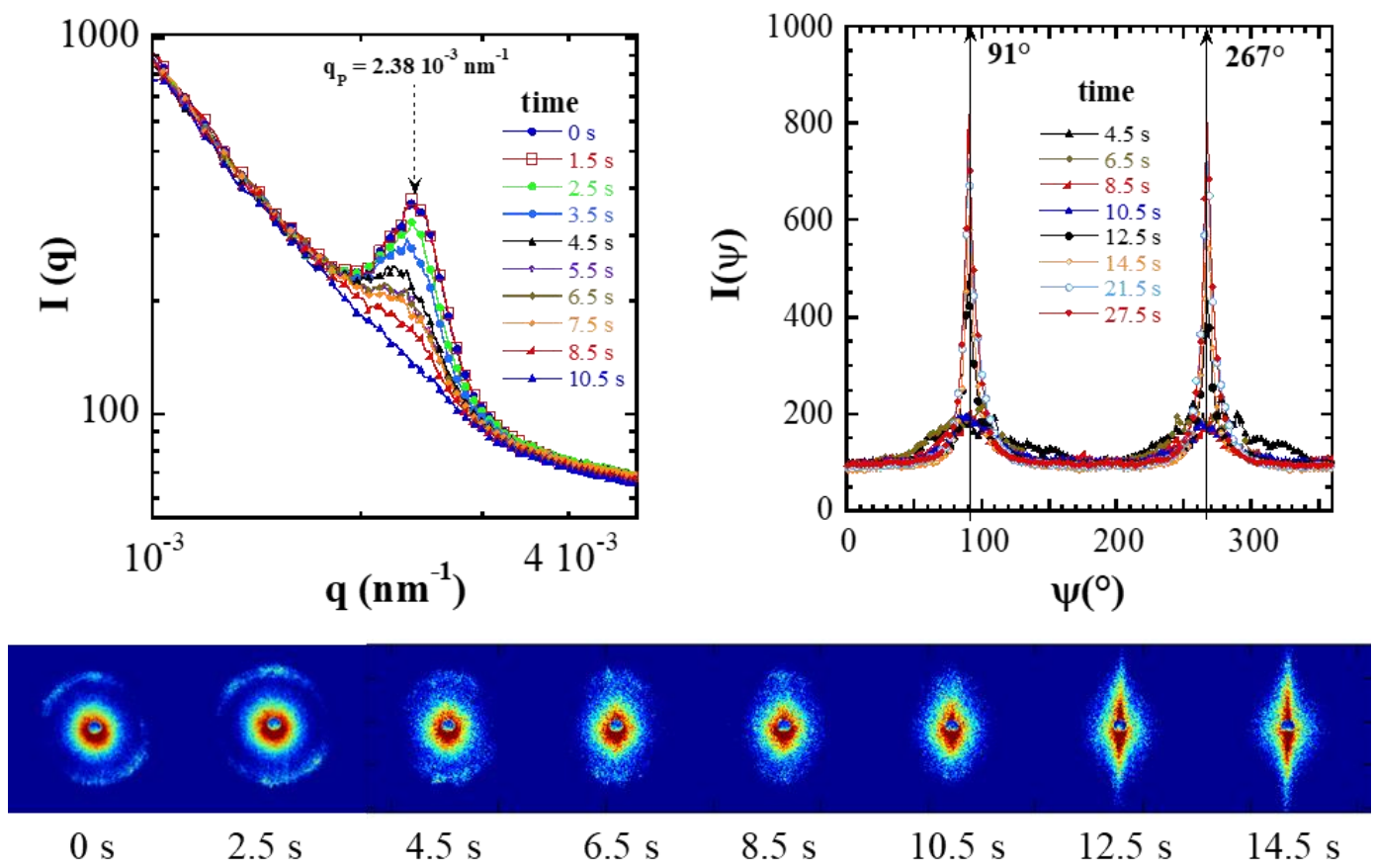
c) Regime III: shear rate $=443 \mathrm{~s}^{-1}$
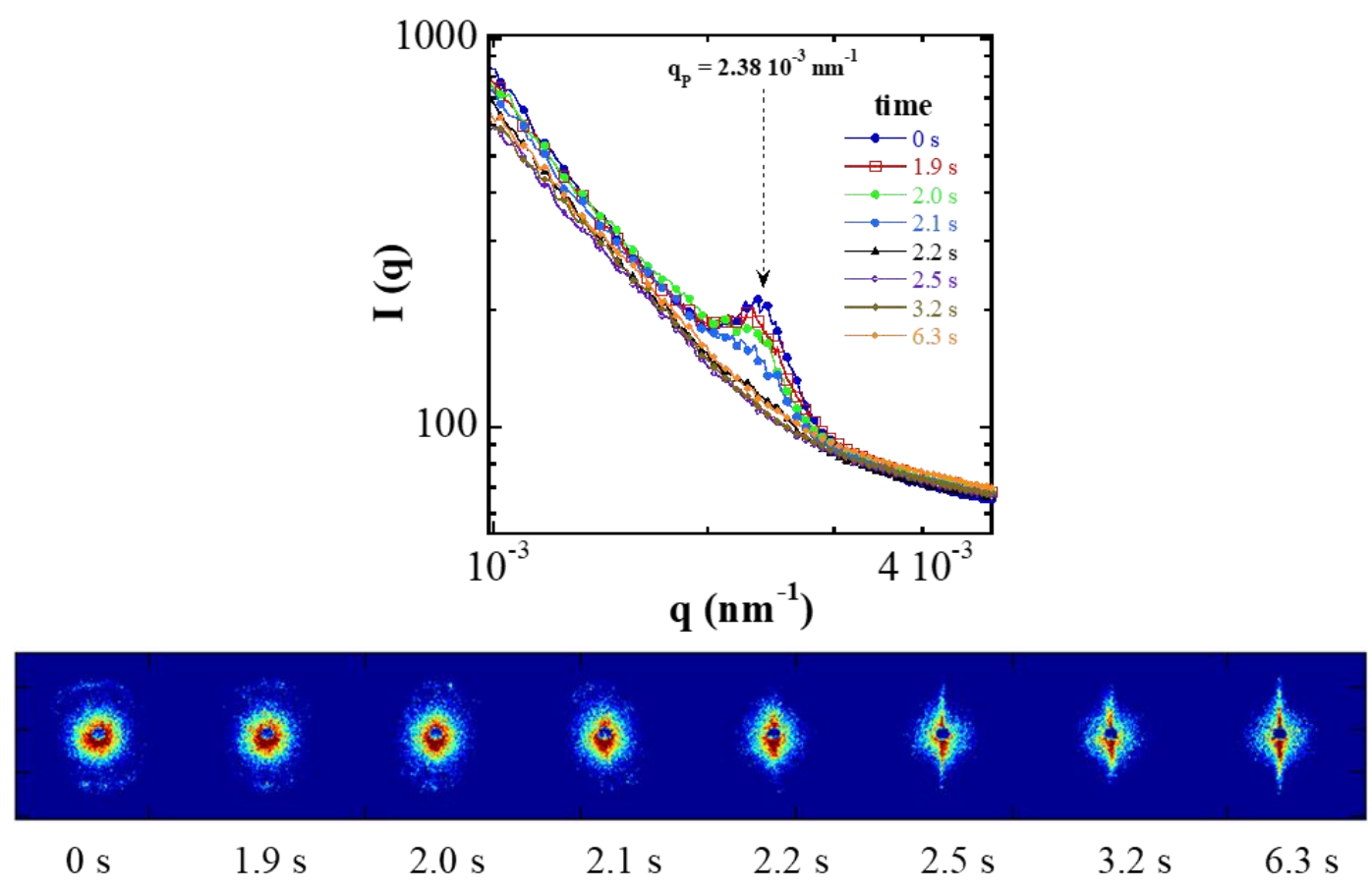

326 Figure 5. Transient state start flow of the CNC suspensions under in situ SALS. Scattering patterns 327 and corresponding azimuthally and radially averaged intensities as a function of time, pertaining 328 to procedure P1 with shear rates corresponding to regimes I (a), II (b), and III (c) for CNC 329 suspensions, $C=9 \mathrm{wt} \%(\phi=5.82 \mathrm{vol} \%)$ with $0.01 \mathrm{~mol} \mathrm{~L}^{-1} \mathrm{NaCl}$ at $T=25^{\circ} \mathrm{C}$.

331 Regime I: when a shear flow of rate $0.29 \mathrm{~s}^{-1}$ (regime I) was applied, the ring-like pattern 332 observed at rest progressively turned into an anisotropic SALS pattern with a peak intensity 333 oriented along the vertical direction (see Fig. 5 a). Both sector and annular averages of the SALS 334 patterns as a function of time confirmed the continuous presence of a correlation peak related to 335 the pitch value and its progressive orientation in the vertical direction, emphasized by the maximum 336 of scattering intensity growing towards $\psi=90^{\circ}(\mathbf{F i g . ~} 5$ a). This evolution was attributed to a gradual 337 orientation with time of the cholesteric phase under flow with the helical axes oriented vertically. 338 A direct in situ observation of the mutual orientation of the cholesteric domains with their helical 
339 axes oriented in the vertical direction (perpendicular to the velocity direction) and the conservation

340 of the pitch distance assessing the non-breakup of the cholesteric phase in this regime, are revealed

341 for the first time. This specific mutual orientation of the cholesteric domains releases some internal

342 stress inside the suspension that explains the decrease in viscosity in this first shear thinning regime

343 I. Another remarkable feature of these SALS patterns is that towards the lowest vectors $\left(q=10^{-3}\right.$

$344 \mathrm{~nm}^{-1}$ ) the scattering intensity became anisotropic under shear with a degree of anisotropy increasing

345 with time as depicted by the increase in the $I_{v}$ curve as a function of time, until a stable orientation 346 was reached (see Fig. 5a).

347 Regime II: For a shear rate of $13.3 \mathrm{~s}^{-1}$ shown in Fig. $5 \mathbf{b}$ and Fig. S4, as for the beginning of

348 regime I, the ring-like pattern of the rest step was progressively replaced by an oriented SALS

349 pattern with a peak intensity that became oriented along the vertical direction over time $(t=4 \mathrm{~s})$.

350 The position of the ring corresponds to a pitch value of $5280 \mathrm{~nm}\left(q_{\mathrm{P}}=2.3810^{-3} \mathrm{~nm}^{-1}\right)$. Then, the

351 scattering peak corresponding to the initial pitch value progressively weakened and disappeared

352 after $t=10.5 \mathrm{~s}$. At the center of the SALS pattern, the intensity distribution progressively evolved

353 from an isotropic to anisotropic pattern, that became stable when the steady state was reached. Like

354 at the beginning of regime I, this evolution of the SALS patterns was attributed to a progressive

355 orientation of the cholesteric phase under flow, with the helical axis oriented vertically. However,

356 at the higher shear rates of regime II, the disappearance of the pitch signature was well evidenced,

357 which could be interpreted as a progressive disruption of the tactoids in smaller units, whose final

358 size - smaller than the pitch distance - was no longer detectable. This important result clarifies that

359 has been proposed in the literature, which mainly attributed the viscosity plateau regime to the

360 orientation of cholesteric domains along the shear direction and their eventual breakdown (Shafiei-

361 Sabet al., 2012; Derakhshandeh et al., 2013; Shafiei-Sabet et al., 2014; Haywood et al., 2017). In 
362 this study, the in situ SALS data collected under shear have clearly shown that the orientation of

363 the cholesteric domains occurred in regime I, while in regime II, the progressive disruption of the

364 tactoids towards a critical micrometer size lower than the pitch distance would be a possible

365 explanation. The interpretation of the orientation of all the cholesteric domains, originally more

366 attributed to regime II by previous works (Shafiei-Sabet al., 2012; Derakhshandeh et al., 2013;

367 Shafiei-Sabet et al., 2014; Haywood et al., 2017) was shifted to regime I based on the current

368 interpretation of SALS measurements.

369 Regime III: At a shear rate of $443 \mathrm{~s}^{-1}$, the ring-like pattern of the rest step was rapidly replaced

370 by an oriented SALS pattern followed by a progressive decrease of the scattering intensity until the

371 disappearance of the peak as shown in Fig. 5 c. These observations are in accordance with a

372 complete breakdown of the cholesteric micrometer sizes tactoids, and emphasize that at this

373 micrometer lengthscale, there is no more structural orientation or organization like the one

374 observed in regime II. This is in complete agreement with previous literature that proposed the

375 merger of liquid crystalline and isotropic domains into a single domain aligned along the flow

376 direction, yielding a uniform texture when observed using polarized light optical microscopy

377 (Shafiei-Sabet al., 2012, 2014; Haywood et al., 2017).

\subsubsection{Structural Organization at Nanometer Lengthscale: in situ Rheo-SAXS}

379 Rheo-SAXS measurements were performed on samples within a concentration range covering 380 the first two domains of the phase diagram. Two samples of concentrations $\mathrm{C}=1.35 \mathrm{wt} \%(0.84$ $381 \mathrm{vol} \%)$ and $\mathrm{C}=6 \mathrm{wt} \%$ (3.783 vol\%) belonged to the purely isotropic phase. Another two samples 382 belonged to concentration domain corresponding to co-existence of isotropic and chiral nematic 383 phases: $\mathrm{C}=9 \mathrm{wt} \%(5.82 \mathrm{vol} \%)$ and $\mathrm{C}=10 \mathrm{wt} \%$ (6.49 vol\%) for which complex changes in 384 viscosity as a function of shear rate have been reported in the literature (Orts et al., 1998; Shafiei- 


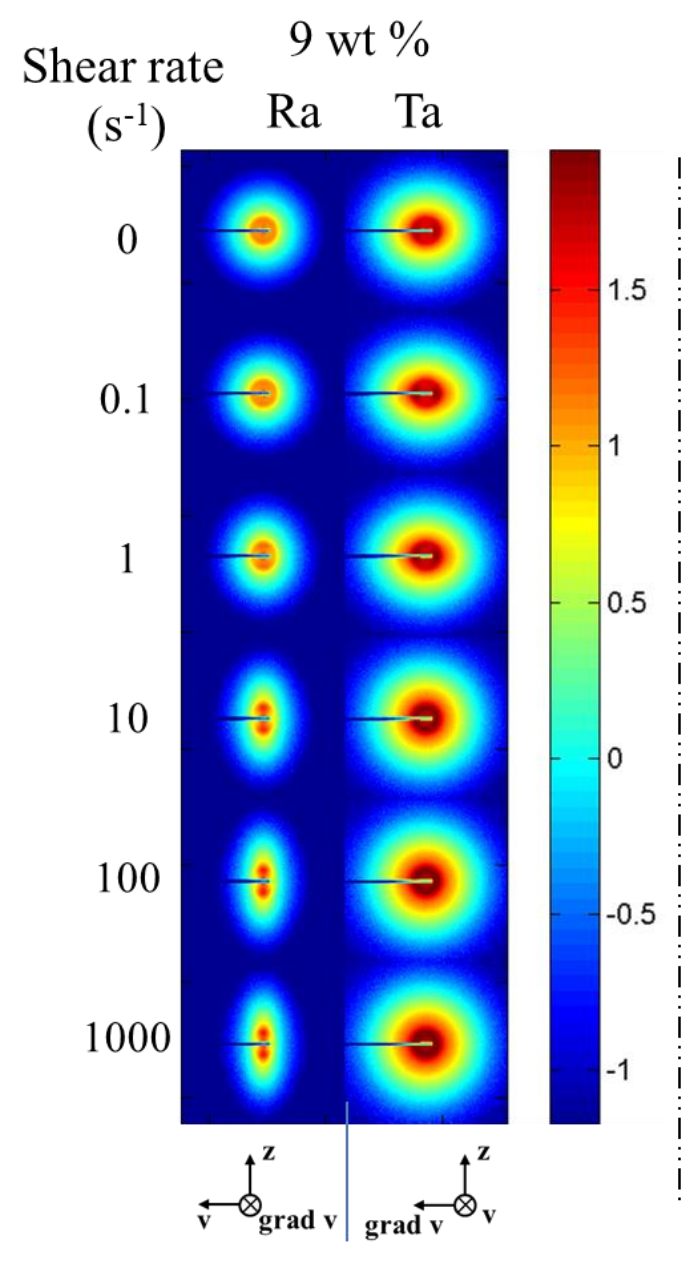

Shear rate $10 \mathrm{wt} \%$

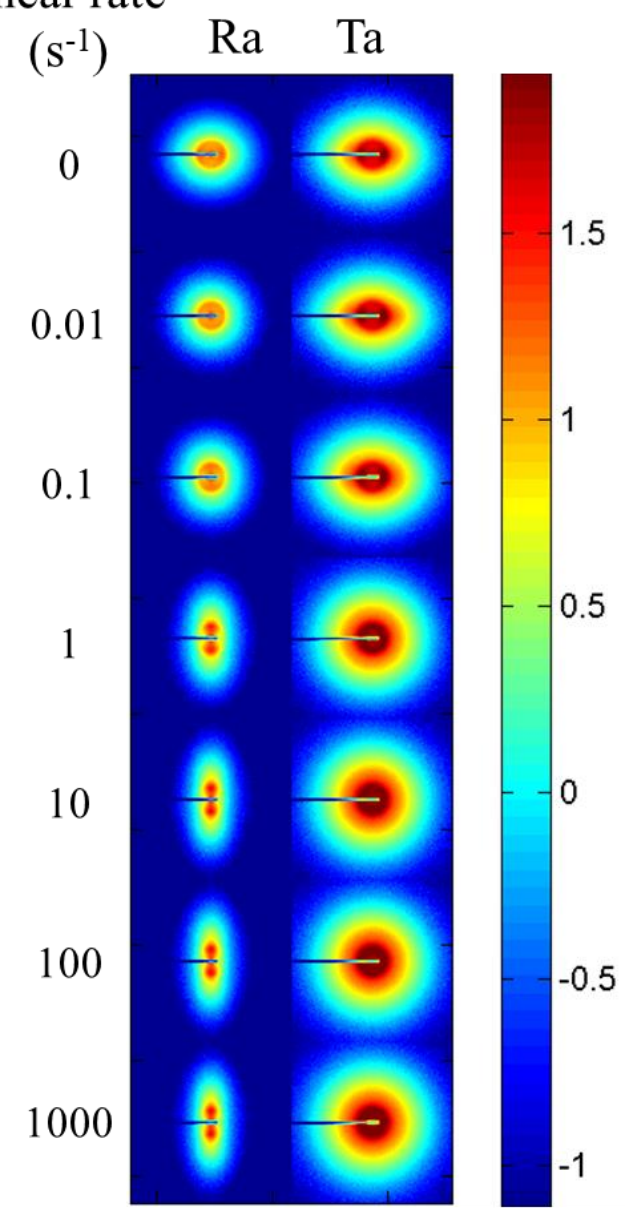

389 Figure 6. 2D-SAXS patterns from a CNC suspension under steady-state flow for successive steps

390 of applied shear rates, in radial ( $\mathrm{Ra}$ ) and tangential (Ta) positions, corresponding to observation

391 planes $(z, v)$ and $(z, \operatorname{grad} v)\left(C=9 \mathrm{wt} \%\right.$, and $C=10 \mathrm{wt} \%, D=2 \mathrm{~m}, 0.01 \mathrm{~mol} \mathrm{~L}^{-1} \mathrm{NaCl}, T=24.5$

$\left.392{ }^{\circ} \mathrm{C}\right)$.

393 SAXS measurements were carried out in radial ( $\mathrm{Ra}$ ) and tangential (Ta) configurations to better

394 describe the orientation of the CNCs in the flow field imposed by the two coaxial cylinders of the

395 shear cell as shown in Fig. 1. The analysis of the anisotropy was performed by using the MATLAB- 
396 based Small-Angle Scattering Evaluation Tool (SASET) software (Muthig et al., 2013).

397 Specifically, the model-free Principal Component Analysis (PCA) method was chosen, which 398 provides values from 0 (for isotropic suspensions) to 1 (in the case of a completely aligned system).

399 On a simulated scattering experiment patterns from a collection of independent differently400 oriented oblate ellipsoids of revolution, the anisotropy PCA parameter showed a good agreement 401 with the more commonly used P2 second order parameter (Muthig et al., 2013). The advantage of 402 anisotropy PCA is that it can be defined on a wide $q$-region, is not dependent of a structure-factor 403 (as the one always found in the CNC SAXS patterns associated to the well-defined mean 404 interparticle distance $d$ between $\mathrm{CNCs}$ ), and its computation does not require additional inputs such 405 as simulated totally aligned scattering patterns (Muthig et al., 2013). The anisotropy and direction 406 of maximum scattering $\left(\psi_{0}\right)$ were calculated via PCA in the $(0.15-2.4) \mathrm{nm}^{-1} q$-range and $(0.04$ $4070.48) \mathrm{nm}^{-1} q$-range at sample-to-detector distances $D=2 \mathrm{~m}$ and $10 \mathrm{~m}$, respectively. One should 408 note that this maximum scattering intensity $\left(\psi_{0}\right)$ can only be defined in the case of anisotropic 409 SAXS patterns and is not defined in the case of isotropic patterns.

410 The 2D-SAXS patterns of CNC suspensions under shear in steady state conditions are presented 411 in Fig. 6 and Figs S6, S7 in radial and tangential modes of observation. The corresponding 412 calculated anisotropy parameter and direction of maximum scattering $\left(\psi_{0}\right)$ were plotted in Fig. 7a 413 and $\mathbf{7 b}$, respectively. For comparison the corresponding viscosities as a function of the shear rate 414 are also plotted in Fig. 7a. 


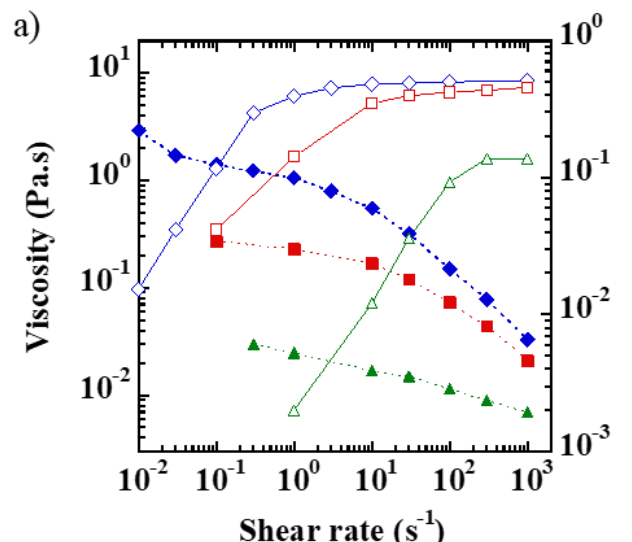

-... Viscosity $10 \mathrm{wt} \%$

- ... Viscosity $9 \mathrm{wt} \%$

Viscosity 6 wt $\%$

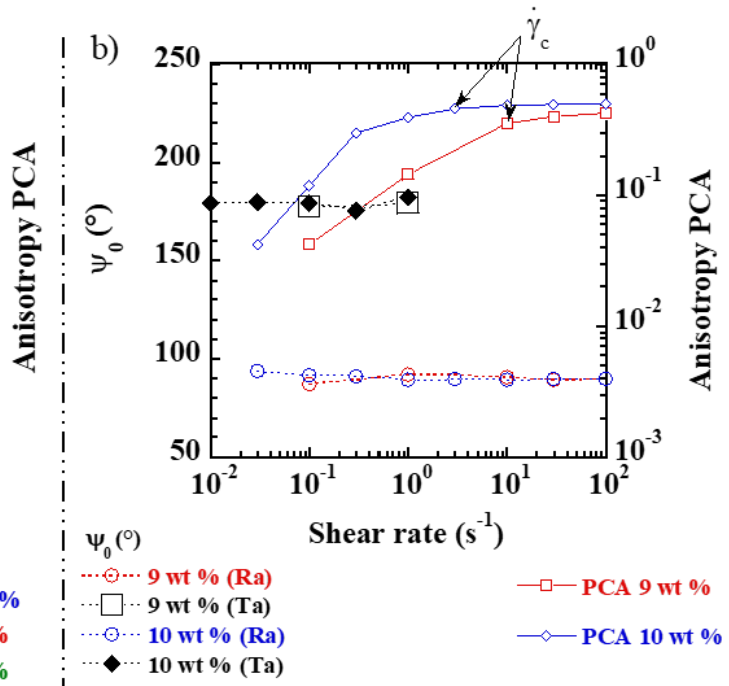

416 Figure 7. Steady-state flow curves (viscosity as a function of shear rate) of CNC suspensions

417 and corresponding PCA anisotropy parameter (a) and direction of maximum scattering $\psi_{0}$

418 (b) calculated from SAXS patterns recorded at different shear rates. The critical shear rate, $\dot{\gamma}_{c}$

419 defines the limit between regimes II and III. ( $\left.0.01 \mathrm{~mol} \mathrm{~L}^{-1} \mathrm{NaCl}, T=24.5^{\circ} \mathrm{C}\right)$.

420 For the lowest concentration of $6 \mathrm{wt} \%$, corresponding to the complete isotropic phase,

421 Anisotropy PCA regularly increased reaching a plateau at the highest applied shear rates. In the

422 second shear-thinning domain, the increase in anisotropy was mainly attributed to the progressive

423 orientation of all isolated CNCs. For the two highest concentrations at 9 and $10 \mathrm{wt} \%$, both in the

424 co-existing isotropic and cholesteric phase domains, anisotropy PCA increased more sharply in the

425 viscosity plateau region (regime II) until reaching a stable value in the second shear thinning range

426 (regime III) Fig. 7a. This type of behavior has previously been observed with CNCs from different

427 origins by rheo-SANS, where a steep increase in the calculated order parameter followed by a

428 plateau above a certain shear rate has been observed (Orts et al., 1998; Haywood et al., 2017). This

429 evolution of the order, or anisotropy parameter, agrees with the theory proposed by Onogi and

430 Asada (1980) in which domains start to break up in the intermediate viscosity plateau domain

431 (regime II), whereas individual rods are free to align in the second shear thinning region (regime 
432 III). Present data coherently follow the same trends: the sharp increase would be attributed to the

433 breakdown of the tactoids as evidenced by SALS at larger lengthscales and the subsequent 434 reorientation of the CNCs reinforced by the orientation of the isolated CNCs in the isotropic phase.

436 The evidence of the disruption of the micrometric tactoids in regime II observed by SALS, is 437 reinforced by the quantitative analysis of the rheo-SAXS measurements performed in radial and 438 tangential modes of observation. In radial mode of observation for the two highest concentrations 439 at 9 and $10 \mathrm{wt} \%$, an increase of scattering intensity is observed in the vertical direction $(z)$ with 440 increasing shear rate (Fig. 6 and Figs S6, S7), corresponding to direction of maximum scattering $441 \psi_{0}$ of about $90^{\circ}$ (Fig. 7b). This increase of scattering intensity can be attributed to a partial 442 alignment of the CNCs with their director along the y direction or velocity $v$. It is due to the 443 reinforcement of fluctuations in particle concentrations along $z$ generated by the alignment of the 444 CNCs along $v$. On the contrary, in tangential mode of observation, an increasing anisotropy of the 445 SAXS patterns along the grad $v$ direction is clearly seen until the critical shear rate, $\dot{\gamma}_{c}$ (Figs. 6 and 446 7b). Indeed, a $\dot{\gamma}_{c}$ of about $10 \mathrm{~s}^{-1}$ at $9 \mathrm{wt} \%$ or $3 \mathrm{~s}^{-1}$ at $10 \mathrm{wt} \%$ belonging to the end of regime II or 447 beginning of regime III in Figs. 6 and 7b, demarcates two specific features of the SAXS patterns. 448 Above $\dot{\gamma}_{c}$, the SAXS pattern is isotropic. Below $\dot{\gamma}_{c}$, the SAXS patterns in tangential mode of 449 observation are elongated along grad $v$ corresponding to direction of maximum scattering $\psi_{0}$ of 450 about $180^{\circ}$ (Fig. 7b). This feature is well in accordance with some elongated micrometer tactoids 451 flowing and oriented along the velocity $v$ as suggested by the SALS analysis and as already 452 observed by rheo-SAXS on flowing clay sepiolite fibers in the same tangential mode of observation 453 (Pignon et al., 2009). The increase of intensity along the grad $v$ direction is due to the reinforcement 454 of fluctuations in the elongated micrometer tactoids oriented along $v$. This orientation of elongated 
455 tactoids under shear has also been proposed by Park et al. (2014). Above $\dot{\gamma}_{c}$, the SAXS patterns in

456 tangential mode of observation became isotropic with an annular form. This is consistent with a

457 progressive breakdown of the micrometer tactoids elongated in smaller units which disintegrate

458 and melt to isolated CNCs at the highest shear rate. This observation is also in agreement with a

459 huge decrease of the scattering intensity in SALS until the disappearance of significant scattering

460 from the sample at the highest shear rates in regime III. These observations are also supported by

461 recent works (Rosén et al., 2020) which studied in detail the projected flow-induced orientation

462 distributions of dispersed CNCs in a square channel flow using scanning microbeam SAXS. From

463 the good agreement between simulations giving the orientation distribution function, and SAXS

464 measurements, they show that at low flow rates, CNCs are highly aligned, indicating that particle-

465 particle interactions inhibit the effects of Brownian rotational diffusion and collectively respond to

466 the shear. They concluded that for semidilute concentration domains, CNCs move collectively into

467 large ellipsoidal tactoids, which themselves have a lower aspect ratio than individual particles and

468 thus spend less time aligned along the flow direction.

469 At highest velocity gradients, when leaving the viscosity plateau to reach the second shear 470 thinning region, above $\dot{\gamma}_{c}$ the anisotropy PCA stabilizes after reaching a maximum level towards 471 an equilibrium value. This stabilization of the orientation level is consistent with the fact that in 472 this region all nanocrystals (irrespective of the phase they initially belonged to) flow in parallel, 473 like in a nematic arrangement and allow the system to flow more easily while maintaining an 474 identical orientation of each crystal. The evolution of the anisotropy PCA as a function of shear 475 rate and concentration emphasized that an optimal alignment was reached when CNCs in chiral476 nematic domains evolve towards a nematic organization. 
Another interesting feature is that the position of the maximum of intensity of the azimuthal

478 averaged (Fig. S5a) or sector (Fig. S5b) averaged scattering intensity occurs at the same $q=q_{\max }$

$479=0.2 \mathrm{~nm}^{-1}$ associated to a well-defined interparticle distance of about $d=2 \pi / q_{\max }=31.4 \mathrm{~nm}$ (at $C$

$480=9 \mathrm{wt} \%$ ), regardless of the applied shear rate and whether the observation is made in radial or

481 tangential mode. The same features have been observed in the past using CNCs from different

482 botanical sources (Orts et al., 1998). This clearly indicates the typical behavior of a flowing liquid

483 crystal, for which objects remain at a well-defined mean distance. Moreover, above $\dot{\gamma}_{c}$, the

484 scattering pattern in the tangential configuration remained isotropic. This shows that the CNCs

485 were oriented along horizontal current lines without adopting any particular organization

486 perpendicular to the flow (no higher order peaks or hexagonal packing). This corresponds to a

487 uniaxial orientation of the particles with a strong stabilization of the orientation of these objects.

488 These results on the structural organization of CNCs under shear are different from those observed

489 for disk-shaped beidellite clay systems (Bihannic et al., 2010, Philippe et al., 2011) or sepiolite

490 clay fibers (Pignon et al., 2009). In these latter cases, the anisotropy of SAXS patterns showed

491 different elongations in radial and tangential observations, which was attributed to a biaxial

492 orientation of the particles.

3.2.3 Complete Description of the Global Structural Organization Pertaining to the threeregime-behavior (Combined SALS and SAXS data)

496 For the three regimes, the steady-state measurements obtained with procedure P2 have allowed 497 confirming the general behavior depicted in start-flow experiments with procedure P1. As shown 498 in Figs. S3, S4, S8, S9, the nanometric CNCs were oriented under flow until large micrometer 499 lengthscales corresponding to the $q$-range (from $\mathrm{L}_{\mathrm{y} 1}=2 \pi / \mathrm{q}_{\mathrm{y} 1}=15.7 \mu \mathrm{m}$ to $\mathrm{L}_{\mathrm{y} 2}=2 \pi / \mathrm{q}_{\mathrm{y} 2}=1.05 \mu \mathrm{m}$ ) 500 explored by SALS. From SALS and SAXS data collected within a shear rates range from $10^{-2}$ to 
$50110^{3} \mathrm{~s}^{-1}$ and $\mathrm{CNC}$ concentration in the co-existence region of the phase diagram $(9 \mathrm{wt} \%)$, in

502 conjunction with the viscosity/shear rate rheometric measurements, a complete description of this

503 flow curve with the well-known three-regime behavior can be obtained. The global structural

504 organization pertaining to the three-regime-behavior is schematized in Fig. 8. The first shear-

505 thinning part in regime I at the lowest shear rates, corresponds to a progressive disruption of the

506 liquid crystalline domains to form micrometer sized tactoids aligned towards the velocity direction

507 with an internal cholesteric organization of the CNCs and the helical axes oriented vertically

508 (evidenced by SALS and the presence of the pitch in vertical direction). Upon reaching the

509 viscosity plateau, in the intermediate shear rate domain (regime II), the SALS pattern shows the

510 breakdown of the tactoids into smaller units down to a critical size smaller than the pitch value.

511 The mean anisotropy parameter calculated from the SALS patterns exhibit a maximum value in

512 this plateau regime II, attributed to a maximum of the alignment of these smaller units of tactoids

513 along the velocity direction in this regime. The SAXS measurements confirm the orientation of the

514 smaller units of tactoids in accordance with the elongated anisotropy of the SAXS patterns along

515 the grad $v$ direction in tangential mode of observation, associated to the huge increase of anisotropy

516 PCA. None of these measurements allow to discriminate two possible CNC organizations within

517 the smaller and smaller tactoids. This would be either some CNCs still associated in helical

518 arrangement but with a size lower than the initial pitch of the suspension at rest, or the unwinding

519 of the cholesteric organization towards a nematic-like arrangement as reported in previous works

520 (Gray et al. 2016; Schütz et al. 2020), or even a combination of this two. The third shear-thinning

521 part at the highest shear rates (regime III) corresponds to the complete breakdown of the smaller

522 units of tactoids as evidence by SALS where no significant scattering intensity is detected. The

523 SAXS observations at nanometer lengthscale display a stabilization of the orientation level 
524 consistent with the parallel flowing of all nanocrystals along the velocity direction like into a 525 nematic arrangement.

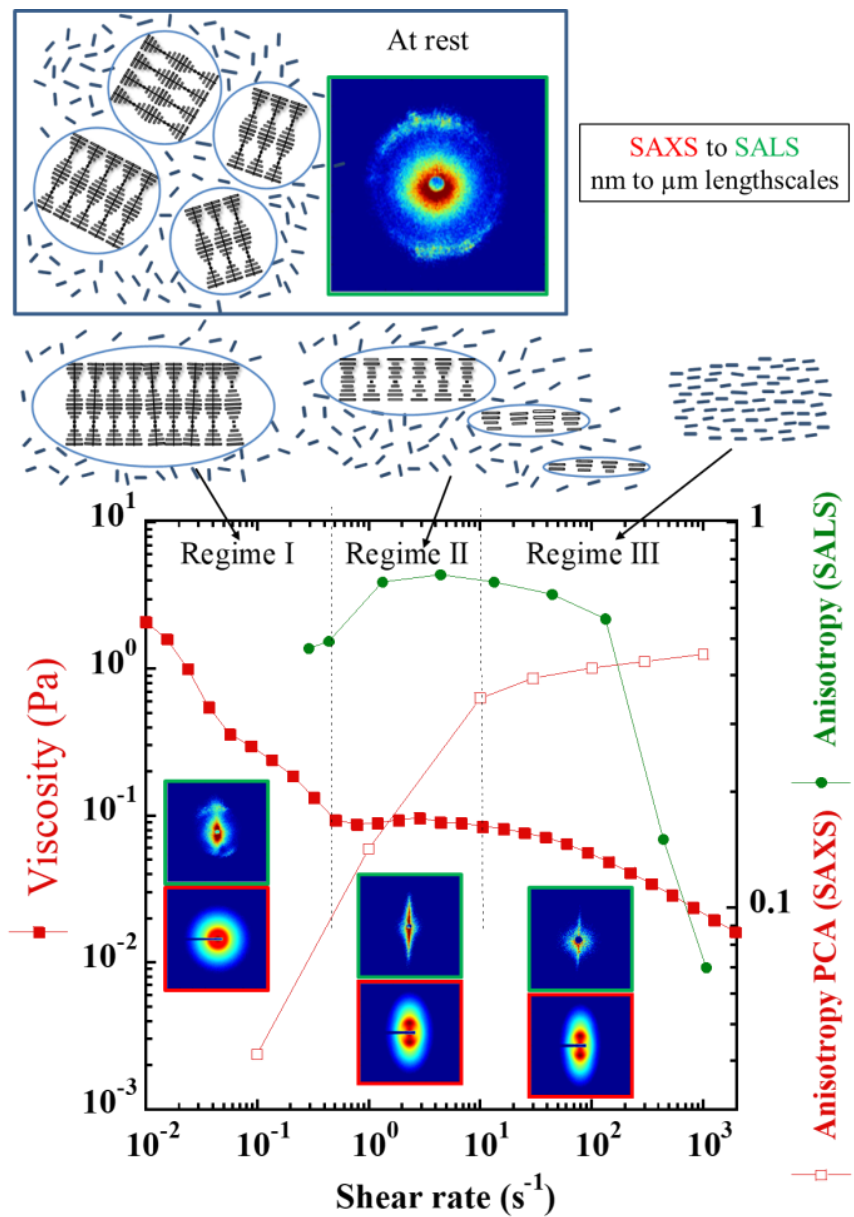

527 Figure 8. Schematic description of CNC suspensions from rest to increasing applied shear rates,

528 displaying transitions from isotropically distributed "cholesteric tactoids" at rest, to aligned

529 "cholesteric tactoids" in regime I, to fragmentation of micrometer size tactoids in regime II, to

530 isolated oriented CNCs in regime III. Viscosity/shear-rate curve from rheometric measurements,

531 anisotropy parameter deduced from SAXS and SALS measurements. Corresponding in situ SAXS

532 and SALS patterns $\left(C=9 \mathrm{wt} \%, 5.82 \mathrm{vol} \%, 0.01 \mathrm{~mol} \mathrm{~L}^{-1} \mathrm{NaCl}\right.$ at $\left.T=24.5{ }^{\circ} \mathrm{C}\right)$. Blue circles or blue

533 ellipses drawn in the scheme identify the cholesteric phase domains or tactoids with respect to the

534 isotropic phase. 
536 3.4. Buildup Mechanisms of CNC Suspensions After Cessation of Flow

537 Two observations were carried out to elucidate the build-up phenomena after a high shear.

538 First, at nanometer scale by rheo-SAXS: after a high shear at $1000 \mathrm{~s}^{-1}$, the viscosity and SAXS

539 patterns were recorded over time under a very low $10^{-2} \mathrm{~s}^{-1}$ shear rate. This allowed having a torque

540 measurement to follow the evolution of viscosity and correlate it with SAXS measurements during

541 relaxation and return to equilibrium of the system under a low shear rate (Fig. 9). Two kinetics of

542 relaxation were identified corresponding to two marked changes in viscosity and PCA parameter

543 evolutions. On the viscosity plot, a first relaxation regime of the order of $700 \mathrm{~s}$ (around $12 \mathrm{~min}$ )

544 was followed by a second longer relaxation regime, established on time scales of $30 \mathrm{~min}$. The

545 anisotropy as quantified by PCA perfectly followed these two processes with a first marked

546 decrease in anisotropy over the first $700 \mathrm{~s}$, followed by a second, slower kinetics with a decrease

547 in anisotropy over time on longer timescales of about $30 \mathrm{~min}$.

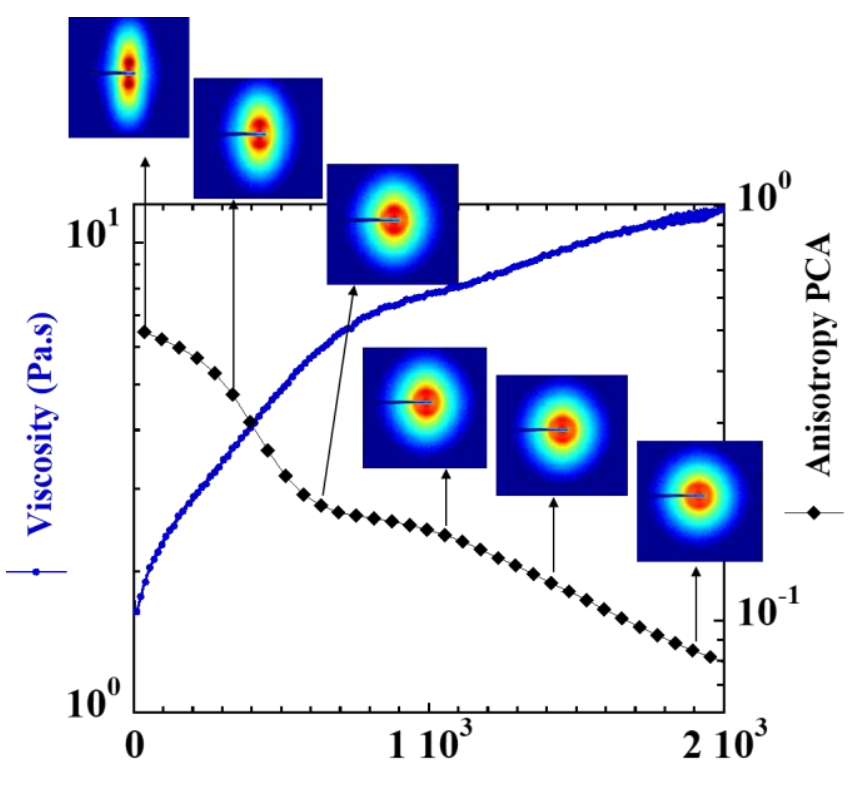

Time (s) 
550 Figure 9. Relaxation process of a CNC suspension under shear of $10^{-2} \mathrm{~s}^{-1}$ after pre-shear at $1000 \mathrm{~s}^{-1}$.

551 Time dependent evolution of viscosity and corresponding anisotropy PCA parameter, from the 552 simultaneously recorded SAXS patterns $(C=10 \mathrm{wt} \%, 6.49 \mathrm{vol} \%$ with sample-to-detector distance $553 D=2 \mathrm{~m}$ and $\left.T=24.5^{\circ} \mathrm{C}\right)$.

554 Second, at large lengthscales by SALS according to procedure P3: after a high shear rate of 1068

$555 \mathrm{~s}^{-1}$ the shear was stopped and the SALS patterns were registered as a function of time (Figs. 10 and 556 S10, S11).

557 From SALS measurements, when a shear rate of $1068 \mathrm{~s}^{-1}$ was applied, no significant scattering 558 signal induced by structural organization was detected. This suggests that CNCs were flowing 559 independently, with an orientation that was established only at the CNC nanometer scale as shown 560 by SAXS data. After cessation of shear flow the relaxation process followed three steps with 561 different dynamics.

562 Step 1: when the shear was stopped, surprisingly, it took only a few seconds for the micrometer563 scale organization to recover, thanks to a fast relaxation process. An anisotropic SALS pattern with 564 an oval shape oriented along the vertical direction was observed at $\mathrm{t}=19 \mathrm{~s}$, which evolved over a 565 few minutes as depicted in Fig. 10a. The corresponding SAXS patterns over this first relaxation 566 step, are also elongated vertically, i.e. perpendicular to the velocity. These multiscale observations 567 are in accordance with the fast build-up of a largescale oriented nematic domain extending from 568 nanometer to micrometer scales. This surprising effect can be attributed to liquid-crystal 569 characteristic behavior, of these colloidal particles, for which the constant interparticle distance as 570 well as the homogeneous interparticle interactions between the crystals promote a rapid re571 organization of the particles. 
572 Step 2: during the following seconds up to first $15 \mathrm{~min}$, the central region of the SALS patterns

573 became progressively isotropic and simultaneously manifested the gradual appearance of a 574 scattered intensity peak along the vertical direction that grew in size and intensity (Fig. 10). At 575 time $675 \mathrm{~s}$ the appearance of the $q_{\mathrm{P}}$ is evidenced. This $q_{\mathrm{P}}$ value corresponds well to that of the 576 dispersion at rest, which is related to the pitch of the cholesteric domains in the sample. It is 577 consistent with the reorganization of cholesteric tactoids (Wang et al., 2016; Wang and 578 MacLachlan 2018; Tran et al., 2018; Liu et al., 2019) with a specific vertical orientation of their 579 helical axis. These observations are supported by recent polarized light microscopy observations 580 (Gray and Mu 2016) who addressed the question of how the texture of samples placed between a 581 microscope slide and cover glass changes with relaxation time when evaporation is inhibited. They 582 observed a transitory nematic-like texture that was induced by shear during the preparation of the 583 sample for polarized light microscopy. From their optical observation, they suggested that there is 584 an alternative mechanism for shear relaxation of $\mathrm{CNC}$ suspensions involving an intermediate stage 585 between nematic and chiral nematic ordered suspensions: a twist-bend-like intermediate and not 586 an untwisting of the chiral nematic phase (Gray and Mu 2016).

587 In parallel to the scattering intensity evolution around this $q_{\mathrm{P}}$, the center of the SALS patterns 588 that was anisotropic and oriented in the vertical direction after a few seconds, slowly became 589 isotropic as shown by the annular integration in Fig. 10b. This evolution at micrometer lengthscale 590 is in good agreement with the rheo-SAXS measurements, emphasizing a fast kinetic of relaxation 591 up to $700 \mathrm{~s}$ for which the anisotropy PCA parameter sharply decreased while a fast increase in 592 viscosity was measured (Fig. 9).

593 This mechanism of relaxation could be associated to a demixing process that corresponds to the 594 isotropic and cholesteric phase separation with an induction time (about $675 \mathrm{~s}$ ). Letting et al. (2005) 
595 have studied in detail the different demixing process of the nematic-isotropic phase transition of

596 rodlike colloids after a quench from the flow-induced fully nematic state. They highlighted that the

597 two mechanisms of phase separation (spinodal decomposition and nucleation-and-growth) can be

598 distinguished during the initial stages of phase separation from (i) the difference in morphology

599 (interconnected structures versus growth of isolated nuclei) and (ii) the delay time before the phase

600 separation sets in (no delay time for spinodal decomposition and a finite induction time for

601 nucleation-and-growth). The relaxation features evidenced in this work on CNC suspensions

602 clearly exhibit an induction time associated to the appearance of a pitch revealing the formation of

603 cholesteric tactoids, which allowed demonstrating that the most probable mechanism of relaxation

604 is associated to a nucleation-and-growth process.

605 Step 3: Following this continuous relaxation process, a third longer relaxation took place over

606 time up to $1668 \mathrm{~s}$. The annular averages of these patterns during relaxation show a progressive

607 evolution of the angular distribution of the scattered intensity around this peak, over a long

608 timescale as presented in Fig. 10b. After about $30 \mathrm{~min}$, an isotropic distribution is reached, giving

609 rise to a ring-like scattering pattern equivalent to that of a suspension at rest. In the same manner

610 at nanometer lengthscale a slower evolution of the increase of viscosity and decrease of anisotropy

611 parameters is evidenced until $30 \mathrm{~min}$ of relaxation (Fig. 9).

612 

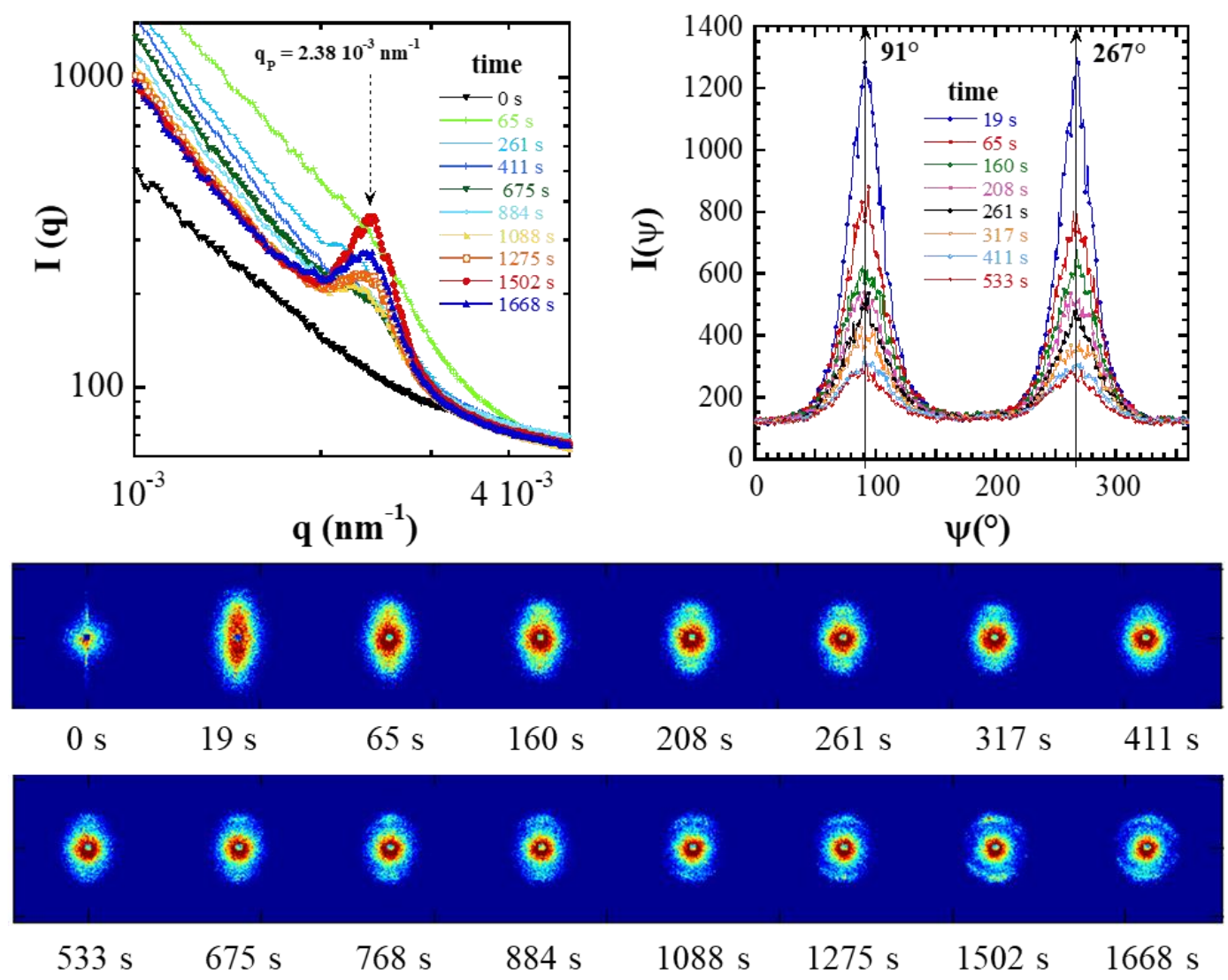

614 Figure 10. Transient state relaxation of the CNC suspensions under in situ SALS. Scattering

615 patterns and corresponding azimuthal (a) and annular (b) average intensities as a function of time,

616 pertaining to procedure P3 with initial shear rates belonging to regime III at $1068 \mathrm{~s}^{-1} . C=9 \mathrm{wt} \%$

$617(\phi=5.82 \mathrm{vol} \%)$ with $0.01 \mathrm{~mol} \mathrm{~L}^{-1} \mathrm{NaCl}$ at $T=25^{\circ} \mathrm{C}$.

\section{4. Conclusions}

620 In this work, the complex three-regime rheological behavior of CNC suspensions under shear

621 flow, typical of liquid crystalline systems, has been studied with a multi-lengthscale approach, in

622 the coexistence regime of isotropic and cholesteric phases, from the size of elementary CNCs (by

623 rheo-SAXS) to that of their assemblies into a cholesteric organization (by SALS under flow). The

624 change in viscosity identified in rheometric measurements has been correlated with structural 
625 parameters over a wide lengthcales (including anisotropy parameters, CNC interparticle and 626 presence or absence of the cholesteric pitch) as a function of time and shear rate, in steady and 627 transient flows (start flow) as well as during the system relaxation after cessation of shear.

628 Thanks to SALS data under shear flow, the changes in chiral nematic organization, and the 629 disappearance and reappearance of pitch under flow and during relaxation, respectively have been 630 described for the first time. The combination with the Rheo-SAXS results in radial and tangential 631 modes of observation clarified the shear-induced structural evolutions and dynamic changes in 632 chiral nematic organization along the well-known three-regions behavior.

633 Several breakthrough have been achieved thanks to this multiscale characterization. First, it has 634 been shown that at lowest shear rates of shear thinning regime I, during the first gradual disruption 635 of large liquid crystalline domains into smaller micrometer-sized tactoids, the cholesteric domains 636 orient with their helical axis aligned perpendicular to the flow direction. This orientation effect had 637 previously been attributed to the plateau regime II (Orts et al., 1998; Shafiei-Sabet al., 2012; 638 Derakhshandeh et al., 2013; Shafiei-Sabet et al., 2014; Haywood et al., 2017). Consequently, this 639 result clarifies what has been previously proposed in the literature, by shifting the current 640 interpretation of the organization of CNCs to lower shear rate ranges.

641 Second, the presence of sub-micrometer sized elongated tactoids flowing and oriented along the 642 velocity have been clearly evidenced in the viscosity plateau regime II, thanks to the joint 643 interpretation of 2D-SAXS and 2D-SALS patterns. The disappearance of the pitch distance in this 644 domain was revealed for the first time, which demonstrates that the size of the micrometer-size 645 elongated tactoids are smaller than the pitch.

646 Third, the buildup mechanisms, upon cessation of the high shear corresponding to regime III 647 have been highlighted for the first time in the pertinent micrometer lengthscale. The appearance of 648 anisotropic SAXS and SALS patterns elongated vertically, i.e. perpendicular to the previous flow 
649 direction, evidenced an impressive fast reassembling of CNCs into a nematic-like organization 650 with a parallel orientation of $\mathrm{CNCs}$ along the previous velocity direction which was characterized

651 on a broad range of lengthscales (from nanometer to micrometer). After that, surprisingly, a slower 652 mechanism, associated to a slower increase in viscosity, was established: this largescale oriented 653 nematic organization evolved into orientated cholesteric micrometric domains with their helical 654 axes aligned perpendicular to the previous flow direction. This relaxation step is associated to a 655 demixing process which corresponds to the isotropic and cholesteric phase separation with an 656 induction time of about $15 \mathrm{~min}$. This suggested that the mechanism of relaxation was probably 657 mainly associated to a nucleation-and-growth process. Finally, a much slower reorganization of 658 these large oriented domains took place towards an isotropic distribution of cholesteric 659 arrangements to build up the final isotropic equilibrium structure of $\mathrm{CNC}$ suspensions at rest.

660 The approach used in this work have shed new light on both particle organization at the relevant 661 lengthscale and structuration mechanisms in CNC suspensions under shear flow. This approach 662 could be applied to better understand buildup mechanisms during nanocomposite elaboration 663 involving shear flow processing, in order to fabricate nanocellulose-based materials with optimal 664 control of the structural and functional properties as conductive, barrier, mechanical or optical 665 properties.

\section{Acknowledgement}

668 We sincerely thank Erwan Paineau (LPS, Saclay) for constructive discussions and William 669 Chèvremont (Laboratoire Rhéologie et Procédés) for his great help in improvement of the SALS 670 set-up. We also thank Jacques Gorini (ESRF, Grenoble), Didier Blésès, Frédéric Hugenell and Eric 671 Faivre (Laboratoire Rhéologie et Procédés) for technical assistance. ESRF is acknowledged for 
672 provision of synchrotron beamtime (proposal SC-4177 and in-house beam time), as well as the

673 ICMG-NanoBio platform (FR 2607, Grenoble) for granting access to the electron microscopy

674 facility. This work has received the financial support of Institut Carnot PolyNat (Investissements

675 d'Avenir - grant agreement \#ANR-16-CARN-0025-01) and was carried out within the framework

676 of the RheoNat and NanoCompUV projects. LRP is part of LabEx Tec21 (Investissements

677 d'Avenir - grant agreement no. ANR-11-LABX-0030) and CERMAV is part of LabEx Arcane and

678 CBH-EUR-GS (Investissements d'Avenir - grant agreement \#ANR-17-EURE-0003). Both

679 laboratories are part of the Glyco@Alps programme (Investissements d'Avenir - grant agreement

680 \#ANR-15-IDEX-02).

681

682

\section{References}

683

Alizadehgiashi, M., Khabibullin, A., Li, Y., Prince, E., Abolhasani, M., Kumacheva, (2018). E. Shear-Induced Alignment of Anisotropic Nanoparticles in a Single-Droplet Oscillatory Microfluidic Platform. Langmuir, 34, 322-330.

Bercea, M., Navard, P. (2000). Shear dynamics of aqueous suspensions of cellulose whiskers. Macromolecules , 33, 6011-6016.

Bihannic, I., Baravian, C., Duval, J.F.L., Paineau, E., Meneau, F., Levitz, P., de Silva, J.P., Davidson, P., Michot, L.J. (2010). Orientational order of colloidal disk-shaped particles under shear-flow conditions: a rheological-small-angle X-ray scattering study. J. Phys. Chem. B, $114,16347-16355$.

Derakhshandeh, B., Petekidis, G., Shafiei-Sabet, S., Hamad, W.Y., Hatzikiriakos, S.G. (2013). Ageing, yielding, and rheology of nanocrystalline cellulose suspensions. J. Rheol., 57, 131148. 
695 Domingues, R.M.A., Gomes, M.E., Rei, R.L. (2014). The potential of cellulose nanocrystals in 696 tissue engineering strategies. Biomacromolecules, 15, 2327-2346.

697 Dong, X.M., Kimura, T., Revol, J.-F., Gray, D.G. (1996). Effects of ionic strength on the 698 isotropic-chiral nematic phase transition of suspensions of cellulose crystallites. Langmuir, 699 12, 2076-2082.

Dufresne, A. (2017). Nanocellulose: From Nature to High Performance Tailored Materials, 2nd ed., Walter de Gruyter GmbH: Berlin/Boston.

Ebeling, T., Paillet, M., Borsali, R., Diat, O., Dufresne, A., Cavaillé, J.-Y., Chanzy, H. (1999). Shear-induced orientation phenomena in suspensions of cellulose microcrystals, revealed by small angle X-ray scattering. Langmuir, 15, 6123-6126.

Eichhorn S. J. Young R.J., and Davies G. D. (2005). Modeling crystal and molecular deformation in regenerated cellulose fibers. Biomacromolecules, 6, 507-513.

Frka-Petesic, B., Radavidson, H., Jean, B., Heux, L. (2017). Dynamically controlled iridescence of cholesteric cellulose nanocrystal suspensions using electric fields. Adv. Mater., 29, 1606208.

709 Gicquel, E., Bras, J., Rey, C., Putaux, J.-L., Pignon, F., Jean, B., Martin, C. (2019). Impact of 710 sonication on the rheological and colloidal properties of highly concentrated cellulose 711 nanocrystal suspensions. Cellulose, 26, 7619-7634.

712 Gray, D.G., Mu, X. (2016). Twist-bend stage in the relaxation of sheared chiral nematic 713 suspensions of cellulose nanocrystals. ACS Omega, 1, 212-219.

714 Haywood, A.D., Weigandt, K.M., Saha, P., Noor, M., Green, M.J., Davis V.A. (2017). New 715 insights into the flow and microstructural relaxation behavior of biphasic cellulose nanocrystal 716 dispersions from RheoSANS. Soft Matter, 13, 8451-8462. 
Jin, Y., Hengl, N., Baup, S., Pignon, F., Gondrexon, N., Sztucki, M., Romdhane, A., Guillet, A.,

718 Aurousseau, (2015). M. Ultrasonic assisted cross-flow ultrafiltration of starch and cellulose 719 nanocrystals suspensions: Characterization at multi-scales. Carbohydr. Polym., 124, 66-76.

720 Kargarzadeh, H., Huang, J., Lin, N., Ahmad, I., Mariano, M., Dufresne, A., Thomas, S., Gałęski, A. 721 (2018). Recent developments in nanocellulose-based biodegradable polymers, thermoplastic

Lagerwall, J.P.F., Schütz, C., Salajkova, M., Noh, J., Park, J.H., Scalia, G., Bergstrom, L. (2014). Cellulose nanocrystal-based materials: from liquid crystal self-assembly and glass formation to multifunctional thin films. Npg Asia Materials, 6, e80.

Lenfant, G., Heuzey, M.-C., van de Ven, T.G.M., Carreau, P.J. (2017). A comparative study of ECNC and CNC suspensions: effect of salt on rheological properties, Rheol. Acta, 56, 51-62.

Lettinga, M.P., Kang, K., Imhof, A., Derks, D., Dhont, J. K G. (2015). Kinetic pathways of the

Liu, Y., Schütz, C., Salazar-Alvarez, G., Bergström, L. (2019). Assembly, gelation, and helicoidal consolidation of nanocellulose dispersions. Langmuir, 35, 3600-3606.

733 Mitov, M. (2017). Chlolesteric liquid crystals in living matter. Soft Matter, 13, 4176-4209.

734 Muthig, M., Prévost, S., Orglmeister, R., Gradzielski, M. (2013). SASET: A program for series 735 analysis of small-angle scattering data. J. Appl. Crystal, 46, 1187-1195.

736 Narayanan, T., Sztucki, M., Van Vaerenbergh, P., Léonardon, J., Gorini, J., Claustre, L., Sever, 737 F.,Morse, J., Boesecke, P. (2018). A multipurpose instrument for time-resolved ultra-small$738 \quad$ angle and coherent X-ray scattering. J. Appl. Crystal., 51, 1-14.

739 Onogi, S., Asada, T. (1980). Rheology and rheo-optics of polymer liquid crystals. In: Astarita G., 740 Marrucci G., Nicolais L. (eds) Rheology. Springer, Boston, MA.127-147. 
741 Orts, W.J., Godbout, L., Marchessault, R.H., Revol, J.-F. (1998). Enhanced ordering of liquid 742 crystalline suspensions of cellulose microfibrils: A small angle neutron scattering study. $743 \quad$ Macromolecules, 31, 5717-5725.

744 Panine, P., Gradzielski, M., Narayanan, T. (2003). Combined rheometry and small-angle X-ray $745 \quad$ scattering. Rev. Sci. Instrum., 74, 2451-2455.

746 Park, J.H., Noh, J., Schütz, C., Salazar-Alvarez, G., Scalia, G., Bergström, L. and Lagerwall, J.P.F. 747 (2014). Macroscopic Control of Helix Orientation in Films Dried from Cholesteric Liquid748 Crystalline Cellulose Nanocrystal Suspensions. ChemPhysChem, 15, 1477-1484.

749 Philippe, A.M., Baravian, C., Imperor-Clerc, M., De Silva, J., Paineau, E., Bihannic, I., Davidson, 750 P.; Meneau, F., Levitz, P., Michot, L.J. (2011). Rheo-SAXS investigation of shear-thinning 751 behaviour of very anisometric repulsive disc-like clay suspensions. J. Phys.-Condens. Matter, $752 \quad 23,194112$.

753 Piau, J.M., Dorget, M., Palierne, J.F. (1999). Shear elasticity and yield stress of silica-silicone 754 physical gels: Fractal approach. J. Rheol., 43, 305-314.

755 Pignon, F., Magnin, A., Piau, J.M., Belina, G., Panine, P. (2009). Structure and orientation 756 dynamics of sepiolite fibers - poly(ethylene oxide) aqueous suspensions under extensional and 757 shear flow, probed by in situ SAXS. Rheol. Acta, 48, 563-578.

758 Qi, W., Yu, J., Zhang, Z., Xu, H-N. (2019). Effect of pH on the aggregation behavior of cellulose 759 nanocrystals in aqueous medium. Mater. Res. Express, 6, 125078.

760 Revol, J.-F., Bradford, H., Giasson, J., Marchessault, R.H., Gray D.G. (1992). Helicoidal self761 ordering of cellulose microfibrils in aqueous suspension. Int. J. Biol. Macromol., 14, 170-172. 762 Rey, C., Hengl, N., Baup, S., Karrouch, M., Gicquel, E., Dufresne, A., Djeridi, H., Dattani, R., Jin, 763 Y., Pignon, F. (2019). Structure, rheological behavior and in situ local flow-fields of cellulose 
nanocrystal dispersions during cross-flow ultrafiltration. ACS Sustain. Chem. Eng., 7, 10679-10689.

766

767

768

769

770

771

772

773

774

775

776

777

778

779

780

781

782

783

784

785

786

Rosén, T, Wang, R., Zhan C., He, H., Chodankar, S., Benjamin, S., Hsiao, B.S. (2020). Cellulose nanofibrils and nanocrystals in confined flow: Single-particle dynamics to collective alignment revealed through scanning small-angle x-ray scattering and numerical simulations. Phys. Rev. E, 101, 032610.

Sanchez-Botero, L., Dimov, A.V., Li, R., Smilgies, D.-M., Hinestroza, J.O. (2018). In situ and realtime studies, via synchrotron X-ray scattering, of the orientational order of cellulose nanocrystals during solution shearing. Langmuir, 34, 5263-5272

Schütz, C., Agthe, M., Fall, A. B., Gordeyeva, K., Guccini, V., Salajková, M., Plivelic, T. S., Lagerwall, J. P. F., Salazar-Alvarez, G., Bergström, L. (2015). Rod packing in chiral nematic cellulose nanocrystal dispersions studied by small-angle X-ray scattering and laser diffraction. Langmuir, 31, 6507-6513.

Semeraro, E.F., Hengl, N., Karrouch, M,, Michot, L.J., Paineau, E., Jean, B., Putaux, J.-L., Lancelon-Pin, C., Sharpnack, L., Pignon, F. (2020). Layered organization of anisometric cellulose nanocrystals and beidellite clay particles accumulated near the membrane surface during cross-flow ultrafiltration: In situ SAXS and ex situ SEM/WAXD characterization. Colloid Surface A, 584, 124030.

Shafiei-Sabet, S., Hamad, W.Y., Hatzikiriakos, S.G. (2014). Ionic strength effects on the microstructure and shear rheology of cellulose nanocrystal suspensions. Cellulose, 21, 33473359.

Shafiei-Sabet, S., Hamad, W.Y., Hatzikiriakos, S.G. (2012). Rheology of nanocrystalline cellulose aqueous suspensions. Langmuir, 28, 17124-17133. 
Siqueira, G., Bras, J., Dufresne, A. (2010). Cellulosic bionanocomposites: A review of preparation, properties and applications. Polymers, 2, 728-765.

Son, Y. (2007). Determination of shear viscosity and shear rate from pressure drop, and flow rate relationship in a rectangular channel. Polymer, 48, 632-637.

Sztucki M., Narayanan T. (2007). Development of an ultra-small-angle X-ray scattering instrument for probing the microstructure and the dynamics of soft matter, J. Appl. Cryst., 40, 459-462.

793 Tang A., Li, J., Li, J., Zhao, S., Liu, W., Liu, T., Wang, J., Liu, Y. A. (2019).

794 Nanocellulose/PEGDA aerogel scaffolds with tunable modulus prepared by stereolithography 795 for three-dimensional cell culture. Biomater. Sci. Polym., 30, 797-814.

796 Thomas, B., Raj, M.C., Athira, K.B., Rubiyah, M.H, Joy, J., Moores, A., Drisko, G.L., Sanchez, C. (2018). Nanocellulose, a versatile green platform: from biosources to materials and their applications. Chem. Rev., 118, 11575-11625.

Tran, A., Hamad, W.Y., MacLachlan, M.J. (2018). Tactoid Annealing improves order in self$800 \quad$ assembled cellulose nanocrystal films with chiral nematic structures. Langmuir, 34 (2), 646801 652.

802 Ureña-Benavides, E.E., Ao, G., Davis, V.A., Kitchens, C.L. (2011). Rheology and phase behavior 803 of lyotropic cellulose nanocrystal suspensions. Macromolecules, 44, 8990-8998.

804 Wang, P., Hamad, W., MacLachlan, (2016). M. Structure and transformation of tactoids in 805 cellulose nanocrystal suspensions. Nat. Commun., 7, 11515.

806 Wang, P.-X., MacLachlan, M.J. (2018). Liquid crystalline tactoids: ordered structure, defective 807 coalescence and evolution in confined geometries. Phil. Trans. R. Soc. A , 376, 20170042.

808 Xu, H.-N., Tang, Y.-Y., Ouyang, X.-K. (2017). Shear-induced breakup of cellulose nanocrystal 809 aggregates. Langmuir, 33, 235-242. 
810 Xu, Y., Atrens, A., Stokes, J.R. (2020). A review of nanocrystalline cellulose suspensions:

811 Rheology, liquid crystal ordering and colloidal phase behaviour. Adv. Colloid Interface Sci., $812 \quad 275,102076$.

813 